\title{
Conserved Serotonergic Background of Experience- Dependent Behavioral Responsiveness in Zebrafish (Danio rerio)
}

\author{
Zoltán K. Varga, ${ }^{1,2}$ Diána Pejtsik, ${ }^{1 \star}$ László Biró, ${ }^{1,2 *}$ Áron Zsigmond, ${ }^{3}$ Máté Varga, ${ }^{3,4}$ Blanka Tóth, ${ }^{5}$ \\ Vilmos Salamon, ${ }^{1}$ Tamás Annus, ${ }^{3}$ Éva Mikics, ${ }^{1}$ and Manó Aliczki ${ }^{1}$ \\ ${ }^{1}$ Laboratory of Translational Behavioural Neuroscience, Department of Behavioural Neurobiology, Institute of Experimental Medicine, 1083, Budapest, \\ Hungary, ${ }^{2}$ János Szentágothai Doctoral School of Neurosciences, Semmelweis University, Budapest, 1085, Hungary, ${ }^{3}$ Department of Genetics, Eötvös \\ Loránd University, Budapest, 1117, Hungary, ${ }^{4}$ Semmelweis University, "Lendület" Nephrogenetic Laboratory, Hungarian Academy of Sciences, 1083, \\ Budapest, Hungary, and ${ }^{5}$ Department of Inorganic and Analytical Chemistry, Budapest University of Technology and Economics, Budapest, 1111, Hungary
}

Forming effective responses to threatening stimuli requires the adequate and coordinated emergence of stress-related internal states. Such ability depends on early-life experiences and, in connection, the adequate formation of neuromodulatory systems, particularly serotonergic signaling. Here, we assess the serotonergic background of experience-dependent behavioral responsiveness using male and female zebrafish (Danio rerio). For the first time, we have characterized a period during behavioral metamorphosis in which zebrafish are highly reactive to their environment. Absence of social stimuli during this phase established by isolated rearing fundamentally altered the behavioral phenotype of postmetamorphic zebrafish in a challenge-specific manner, partially due to reduced responsiveness and an inability to develop stress-associated arousal state. In line with this, isolation differentially affected whole-brain serotonergic signaling in resting and stress-induced conditions, an effect that was localized in the dorsal pallium and was negatively associated with responsiveness. Administration of the serotonin receptor 1A partial agonist buspirone prevented the isolation-induced serotonin response to novelty in the level of the whole brain and the forebrain as well, without affecting catecholamine levels, and rescued stress-induced arousal along with challenge-induced behaviors, which together indicates functional connection between these changes. In summary, there is a consistent negative association between behavioral responsiveness and serotonergic signaling in zebrafish, which is well recognizable through the modifying effects of developmental perturbation and pharmacological manipulations as well. Our results imply a conserved serotonergic mechanism that contextdependently modulates environmental reactivity and is highly sensitive to experiences acquired during a specific early-life time window, a phenomenon that was previously only suggested in mammals.

Key words: anxiety; arousal; plasticity; serotonin; social isolation; zebrafish

Significance Statement

The ability to respond to challenges is a fundamental factor in survival. We show that zebrafish that lack appropriate social stimuli in a sensitive developmental period show exacerbated alertness in nonstressful conditions while failing to react adequately to stressors. This shift is reflected inversely by central serotonergic signaling, a system that is implicated in numerous mental disorders in humans. Serotonergic changes in brain regions modulating responsivity and behavioral impairment were both prevented by the pharmacological blockade of serotonergic function. These results imply a serotonergic mechanism in zebrafish that transmits early-life experiences to the later phenotype by shaping stress-dependent behavioral reactivity, a phenomenon that was previously only suggested in mammals. Zebrafish provide new insights into early-life-dependent neuromodulation of behavioral stress-responses.

Received Sep. 10, 2019; revised Mar. 17, 2020; accepted Mar. 20, 2020.

Author contributions: Z.K.V., D.P., L.B., M.V., E.M., and M.A. designed research; Z.K.V., D.P., L.B., A.Z., B.T., and T.A. performed research; Z.K.V., D.P., L.B., B.T., and V.S. analyzed data; Z.K.V. wrote the first draft of the paper; Z.K.V., D.P., L.B., A.Z., M.V., E.M., and M.A. edited the paper; Z.K.V., E.M., and M.A. wrote the paper.

Z.K.V. was supported by Hungarian Ministry of Human Capacities Grant UNKP-19-3-III-SE-7, European Social Fund, and Semmelweis University Grant EFOP-3.6.3-VEKOP-16-2017-00009. E.M. was supported by Hungarian Scientific Research Fund Grant K125390 and Hungarian Brain Research Program Grant 2017-1.2.1NKP-2017-00002. M.V. was supported by ELTE Institutional Excellence Program Grant 1783-3/2018/ FEKUTSRAT. D.P. was supported by Hungarian Ministry of Human Capacities Grant UNKP-19-2-II-BME-120. The datasets and analysis script generated during the current study are available in the figshare repository. We thank Christina Miskolczi (Budapest, Hungary) for language editing; Máté Tóth (Budapest, Hungary) for sharing comments that greatly improved the study; Sándor Imre Piross and Péter Fehérvári (Department of Biomathematics and Informatics, University of Veterinarian Studies, Hungary for Statistical Consulting); and László Barna and Csaba Pongor (Nikon Microscopy Center at the Institute of Experimental Medicine, Nikon Austria GmbH, and Auro-Science Consulting, Ltd) for kindly providing microscopy support.

The authors declare no competing financial interests.

${ }^{*}$ D.P. and L.B. contributed equally to this work.

Correspondence should be addressed to Manó Aliczki at aliczki.mano@koki.mta.hu or Zoltán K. Varga at varga.zoltan@koki.mta.hu.

https://doi.org/10.1523/JNEUROSCI.2178-19.2020

Copyright $\odot 2020$ the authors 


\section{Introduction}

Coping with environmental threats is essential for the survival and well-being of animals. Contextually adequate emergence and expression of behavioral responses depend on previous experiences, particularly stimuli acquired during sensitive and plastic developmental periods (Chapman et al., 2004; Nederhof and Schmidt, 2012; Haller et al., 2014; Santarelli et al., 2014). While there are numerous plausible explanations regarding the function of this link e.g., preparing the individual for later environmental challenges, the underlying mechanisms are poorly understood. Clinical and preclinical data suggest that the quality of development of neuromodulatory pathways, particularly the formation of the serotonergic system, affects later behavioral phenotype; hence, it transmits the information of the early-life environment to adulthood (Raleigh et al., 1984; Gross et al., 2002; Lehmann et al., 2003; Fone and Porkess, 2008; Lukkes et al., 2009b; Booij et al., 2015). The diverse and context-dependent behavioral effects of serotonin suggest that these pathways, apart from direct modulation, are able to act as general modulators of behavior (Geyer, 1995; Jing et al., 2009; Yokogawa et al., 2012; Lovett-Barron et al., 2017), possibly through the modification of internal states, such as arousal and alertness. State alertness adjusts behavioral reactivity to current environmental demands e.g., the presence of acute stressors, by fine-tuning sensory-motor functions, information processing, or decision-making processes. Despite its fundamental impact on the expression of behavior, there are only a few studies assessing the neuromodulatory basis of general arousal in healthy animals, while the literature of experience-dependent alertness and associated challenge-responding is even sparser.

The zebrafish (Danio rerio) provides an attractive tool to investigate basic vertebrate neurobiology, due to the ease and accessibility of approaches to modify or screen physiological processes (Lieschke and Currie, 2007; MacRae and Peterson, 2015). Particularly, the use of zebrafish has several advantages to study the neural basis of experience-dependent challengeresponding: (1) their relatively simple behavioral repertoire is primarily driven by robust stress responses, including hormonal and neuromodulatory actions (Egan et al., 2009); (2) their CNS possesses homologous anatomic structures and neurotransmitters to that of mammals with highly conserved function but less complex organization (Panula et al., 2010); and (3) they show significant behavioral plasticity indicated by a robust behavioral reorganization occurring during the late larval stage (Lau et al., 2011). The onset of such metamorphosis gives us the opportunity to assess the impact of experiences in potentially sensitive developmental periods on the adult challenge-responding phenotype.

In the current study, we aimed to use the above described unique features of zebrafish and assess how early-life events affect later challenge-responding phenotypes through alterations in 5-HT signaling. First, we aimed to identify a sensitive developmental period that plays a prominent role in shaping adult behavior. We hypothesized that the behavioral metamorphosis at the late larval stage is characterized by environmental reactivity that may serve as a critical period for shaping the individual's later behavioral phenotype. Identifying such a sensitive period, we subsequently subjected zebrafish to the acute presence and/or chronic absence of environmental stimuli (e.g., social isolation) and analyzed different aspects of challenge responsiveness. In order to do this, we conducted tests that represent nonmeaningful stimuli (i.e., an unidentified threat), such as environmental novelty, and meaningful stimuli, such as a social cue. These contexts evoke different internal states and behavioral responses that may be orchestrated by different neuromodulatory actions (for review, see Behrendt, 2011). We also differentiated between novel visual, social visual, and novel but nonvisual stimuli to exclude potential biasing effects of social isolation on sensory perception. Finally, we assessed isolation-induced changes in serotonergic signaling under resting and challenge-induced conditions by applying molecular, histologic, and pharmacological tools. We conducted measurements at whole-brain level and more specifically in limbic forebrain areas, then functionally correlated serotonergic and behavioral effects by the specific pharmacological blockade of 5-HT signaling.

\section{Materials and Methods}

\section{Animals}

Wild-type $(\mathrm{AB})$ zebrafish lines were maintained in the animal facility of Eötvös Loránd University according to standard protocols (Westerfield, 2000). Experimental subjects were male and female animals aged between 8 and 30 days post fertilization (dpf). Fish were maintained in a standard $14 \mathrm{~h} / 10 \mathrm{~h}$ light/dark cycle. Animals were terminated with tricane immediately after each experiment. Feeding of larvae started at $5 \mathrm{dpf}$ with commercially available dry food (a 1:1 combination of $<100 \mu \mathrm{m}$ and $100-200 \mu \mathrm{m}$ Zebrafeed, Sparos) combined with Paramecium. After $15 \mathrm{dpf}$, juvenile fish were fed using dry food with gradually increasing particle size $(200-400 \mu \mathrm{m}$ Zebrafeed, Sparos) combined with fresh brine shrimp hatched in the facility. All protocols used in our study were approved by the Hungarian National Food Chain Safety Office (Permit \#PEI/001/1458-10/2015 and \#PE/ EA/2483-6/2016).

\section{Environmental modifications}

Chronic social isolation of zebrafish was conducted between 14 and 29 dpf. Animals originating from the same spawning were randomly allocated to social rearing or social isolation. Isolated animals were kept in white opaque plastic tanks $(52 \times 35 \times 46 \mathrm{~mm}$, depth $\times$ width $\times$ length $)$ (see Fig. $2 a$ ) depriving the individuals of sensory cues from conspecifics. Tanks were filled with EIII medium, half of the volume of which was replaced on a daily basis. Socially reared control animals were subjected to similar conditions, with the exception of the size of their aquarium, which was matched to the greater number of larvae, providing $\sim 50 \mathrm{ml}$ volume for each larva.

Light/dark tank (LDT) exposure, considered as an acute challenge, was used as a developmental experience as well as a behavioral sampling tool. The procedure was identical in both cases and is described in Behavioral tests and analysis.

\section{Drug treatments}

Buspirone (Sigma Millipore) was dissolved in EIII medium and administered as a water bath for $10 \mathrm{~min}$ followed by a $1 \mathrm{~min}$ washout in compartments of a 24-well plate. Each compartment (15.6 $\mathrm{mm}$ in diameter) contained $1.5 \mathrm{ml}$ of treatment solution. We applied 0 (vehicle) and $50 \mathrm{mg} / \mathrm{L}$ concentrations, which were selected based on our previous studies (Varga et al., 2018).

\section{Behavioral tests and analysis}

For LDT testing, half of the base of a plastic tank $(90 \times 25 \times 24$, length $\times$ width $\times$ depth) (see Fig. $1 b$ ) was masked with opaque matte black paint (dark zone), whereas the other half remained intact, penetrable by light (light zone). Experiments were conducted during the second part of the light phase of the daily cycle, as zebrafish are reported to show continuous activity in this period (MacPhail et al., 2009). Eight subjects were tested simultaneously over the span of a single trial. Experiments in which pharmacological treatments were used began with a 10 min treatment bath in a 24-well plate compartment, after which animals were gently pipetted to another compartment containing EIII medium for a 1 min washout and then were moved to the testing apparatus for $10 \mathrm{~min}$. Behavior of fish was recorded by a digital video camera. Animals were 
a

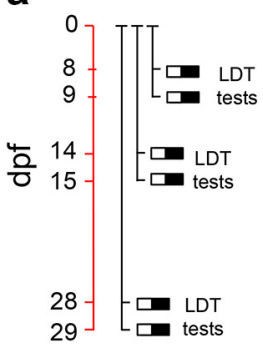

b LDT

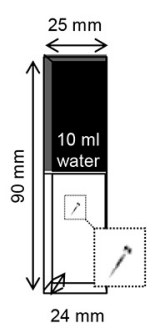

C

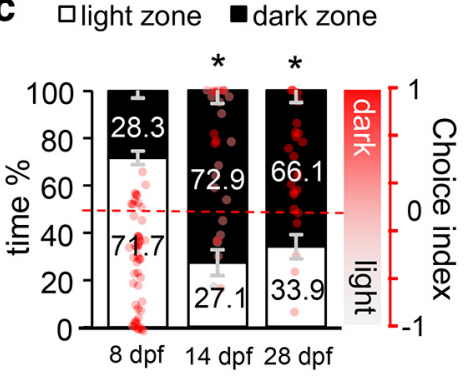

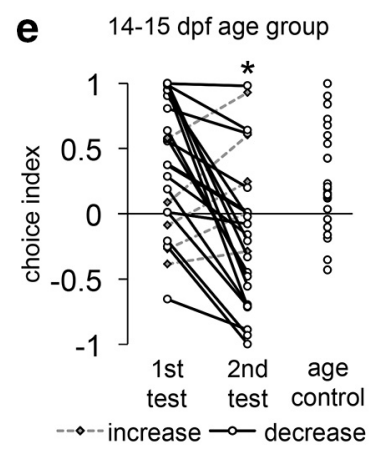

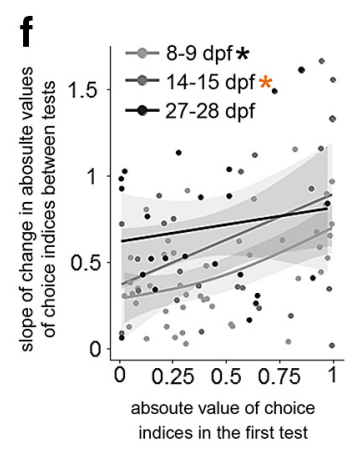

Figure 1. a, Protocol of Experiment 1. Red timeline represents the age of larvae expressed in days post fertilization (dpf). Individual black lines indicate different groups of larvae. $\boldsymbol{b}$, Schematic figure of the LDT apparatus. $\boldsymbol{c}$, Avoidance behavior of zebrafish at the first testing day throughout the first month of development. Left axis indicates the percentage of time. Right axis and red dots represent choice index in which 1 indicates $100 \%$ time in the dark zone, whereas -1 represents $100 \%$ time in the light zone. * Significant difference compared with 8 dpf age group. $\boldsymbol{d}$, Slope of change in avoidance behavior between the 2 testing days. *Significant difference compared with 8 dpf age group. $\boldsymbol{e}$, Individual values of choice indices in the 14-15 dpf age group and test naive age control. Dashed lines indicate increased avoidance. Solid lines indicate decreased avoidance. *Significant difference from first testing day. $\boldsymbol{f}$, Relation between the first and second days of testing. Trends are indicated by solid regression lines and 95\% Cls. *Significant linear regression. Orange asterisk indicates marginal significance $(p=0.0583)$. LDT: light/dark tank.

placed in the light zone of the apparatus directly next to the border of zones. Experimental tanks (see Fig. $1 b$ ) were each filled with $10 \mathrm{ml} \mathrm{me-}$ dium and thoroughly cleaned between trials. To reduce interfering stimuli from the environment, experimental apparatuses were covered with an opaque black plastic box with a hole on top allowing the attachment of the video camera. The apparatuses were illuminated from beneath with white LED panels covered by matte Plexiglas. Video recordings were analyzed using EthoVision XT 12 automated tracker software (Noldus et al., 2001) and Solomon Coder (András Péter) manual tracker software. Time spent in, and transitions between, light and dark zones were measured. Distance swum and mean velocity were measured only in the light zone. To describe scotophobia (dark avoidance) and scototaxis (dark preference), a choice index was defined as the time spent in the dark zone of the compartment relative to time spent in the light zone. Consequently, choice index of 1 indicates $100 \%$ time in the dark zone, choice index of -1 represents $100 \%$ time in the light zone, and choice index of 0 represents $50 \%$ in the dark zone and $50 \%$ in the light zone.

To measure arousal and visual responsiveness of fish, standard, transparent 6-well plates ( $34.8 \mathrm{~mm}$ in diameter) were used. Illumination and visual stimuli were presented by a screen of a tablet PC from beneath. The choice of time for testing and the applied pharmacological treatment protocols were the same as those in earlier experiments. The experiment consisted of two parts: one was considered as a resting and the other was considered as an acute stress-affected period. To establish basal conditions, a $20 \mathrm{~min}$ habituation phase was introduced before the first part, as zebrafish have been reported to habituate to novelty in this amount of time (Yokogawa et al., 2012). To measure behavior in stressaffected conditions, the second part took place immediately after the transfer of animals to a novel compartment. In order to measure arousal state and accompanied sensory responsiveness of the animals, during

both parts a black object was projected onto the bottom of the testing compartment that remained stationary for $5 \mathrm{~min}$, then started to rotate in circles, with its speed increasing twofold in every $2 \mathrm{~min}$ (from 0.5 to $8 \mathrm{rpm}$ ) for an overall $10 \mathrm{~min}$. Both the initial position and subsequent movement directions of the items were randomized among test subjects. The number of active swimming episodes was measured with a custom-made clustering algorithm based on color clustering $(k$ means clustering). We considered a swimming episode active if $>1 \%$ pixel change occurred between two consecutive frames. This threshold was calculated empirically from consecutive frames showing stationary or moving animals.

To measure avoidance responses to nonmeaningful (e.g., novelty-induced, nonvisual challenges), the swimming plus-maze (SPM) test was used, recently developed by our laboratory (Varga et al., 2018). The apparatus is a + -shaped platform consisting of two plus two opposite arms, different in depth, connected by a center zone. Larval and juvenile zebrafish prefer the deep over the shallow arms and the center zone, a preference linked to anxiety-like motivational states. For the validation procedure and detailed specification of the test, see Varga et al. (2018). Ten-minute-long behavioral tests were conducted by using the same protocol as described at the LDT. Time spent in each zone and the mean of overall velocity were measured. To characterize arm preference, a choice index was defined as the relative time spent in the deep compared with the time spent in the shallow arms. Consequently, a choice index of 1 indicates $100 \%$ time in the deep arms, whereas a choice index of -1 represents $100 \%$ time in the more aversive shallow arms, and a choice index of 0 represents an equal amount of time in each arm.

To measure avoidance responses to meaningful (e.g., social visual challenges), the social preference test of the Dreosti laboratory was used (Dreosti et al., 2015). The test apparatus is a Ushaped platform $(40 \times 32 \mathrm{~mm})$ consisting of two identical arms, with glass window partitions enabling only visual communication between zebrafish. The test is based on the visually driven preference of larvae toward conspecifics, a phenomenon emerging around the third week of development. Fifteen-minute-long behavioral tests with the presence of a conspecific were preceded by a fifteen-minute-long habituation phase according to the protocol from the original paper. Time spent in each zone was measured. To characterize social preference, a choice index was defined as the relative time spent in the arm with the conspecific compared with the time spent in the empty arm. Consequently, a choice index of 1 indicates 100\% social preference, a choice index of -1 represents $100 \%$ social aversion, and a choice index of 0 represents no preference or aversion.

\section{High performance liquid chromatography-tandem mass spectrometry (HPLC/MS/MS)}

Zebrafish were terminated and kept at $-80^{\circ} \mathrm{C}$ until measurements. The body and eyes of each subject were manually removed on ice-cold Petri dishes; then tissue samples were pooled from the remains of either two (Experiment 3) or three (Experiment 4) randomly chosen individuals of the same treatment group. The samples were transferred to Eppendorf tubes, and their weight was measured on a semi-micro balance (Precisa, EP 225SM-DR). After the addition of $50 \mu \mathrm{l}$ of $10 \mathrm{~mm}$ ammonium formate, $0.1 \%$ formic acid solution containing $5 \%$ methanol, the samples were homogenized using an ultrasonic sonotrode, and the homogenates were centrifuged to produce protein-free supernatants for HPLC-MSMS analysis. The separation was conducted by HPLC composed of a PerkinElmer series 200 high pressure gradient pump, an autosampler, an 
online degasser, and a thermostat. A Phenomenex Luna Omega Polar C18 $50 \times 3 \mathrm{~mm}, 3 \mu \mathrm{m}$ column was used with a gradient elution with methanol (mobile Phase A) and $0.1 \%$ formic acid (mobile Phase B): $0-0.8 \mathrm{~min}$ : at $3 \% \mathrm{~A} ; 1.3-1.8 \mathrm{~min}$ : at $80 \% \mathrm{~A} ; 2-3 \mathrm{~min}$ : at $3 \% \mathrm{~A} ; 10 \mu \mathrm{l}$ samples were injected, and the flow rate was $500 \mu \mathrm{l} /$ min. Serotonin, dopamine, noradrenaline, and 5-HIAA were detected using a triple quadrupole mass spectrometer (Applied Biosystems, MDS SCIEX 4000 Q TRAP) in positive multiple reaction monitoring mode (MRM transitions: serotonin: 177.2 $\rightarrow$ 170.0, dopamine: $154.1 \rightarrow 137.1$, noradrenaline: $152.2 \rightarrow$ 135.1, 5-HIAA: 192.2 $\rightarrow 146.2$ ).

\section{Immunohistochemisty and confocal microscopy}

Zebrafish were fixed overnight in 4\% PFA in PBS. For the preparation of cryosections, cryoprotecting dehydration of wholebody samples was conducted in a 10\% $20 \%-30 \%$ sucrose-PBS gradient. Samples were subsequently embedded in TissueTek OCT medium, frozen on dry-ice, and stored at $-80^{\circ} \mathrm{C}$ until cryosectioning; $20 \mu \mathrm{m}$ sections were cut in a Microm HM505 cryostat. Sections were collected onto slides (Thermo Fisher Scientific), dried at room temperature overnight, and stored at $-80^{\circ} \mathrm{C}$ until immunohistochemistry was performed.

For immunohistochemistry, slides with sections were bordered with liquid blocker using a 2-mm-wide PAP pen (Merck). For each step, $400 \mu \mathrm{l}$ liquid solution was administered for each slide. Sections were washed 3 times in PBS (10 min each) and incubated overnight at room temperature with polyclonal anti-5-HT antibody produced in rabbit (1:500, 20080, ImmunoStar) diluted in PBS containing 0.5\% BSA (A7906, Sigma Millipore) and $0.25 \%$ Triton X-100 (T8787, Sigma Millipore). Sections were washed three times in PBS and incubated with goat anti-rabbit AlexaFluor-488 (1:500, 111-545-003, Jackson ImmunoResearch Laboratories) for $2.5 \mathrm{~h}$. After several washes in PBS, sections were incubated with DAPI (1:10,000, D8417, Sigma Millipore) for $30 \mathrm{~min}$. Slides were mounted with Mowiol4-88 (81381, Sigma Millipore) fluorescent mounting medium.

Images were taken using a $\mathrm{C} 2$ confocal laser scanning microscope (Nikon Europe) using a $20 \times$ (Plan Apo VC, NA $=0.75$ ) objective $(x y$ : $0.31 \mu \mathrm{m} /$ pixel). We used 405 (Coherent) and 488 (CVI Melles Griot) lasers, and scanning was done in channel series mode. The pixel dwell time, photomultiplier tube gain, laser intensity detector, and pinhole settings were kept constant for all image acquisitions.

Image analysis was conducted using the ImageJ software (Schneider et al., 2012). Standard $30 \times 30 \mu \mathrm{m}$ ROIs were chosen and applied in each investigated area. Individual subtract values of a given image were calculated in a neighboring ROI, containing no fibers, by the enhancement of the subtraction to a level in which the background was totally diminished. Subsequently, chosen values were applied to relevant ROIs, and the percentage of 5-HT signal in a binary processed picture was measured. Individual data were calculated as means of measurements of the two hemispheres.

\section{Experimental design}

In Experiment 1, we aimed to determine the time point of shift from the light- to the dark-preferring phenotype during development, representing a specific aspect of the behavioral metamorphosis, by subjecting different sets of zebrafish to the LDT test for $10 \mathrm{~min}$ at 8,14 , and $28 \mathrm{dpf}$,

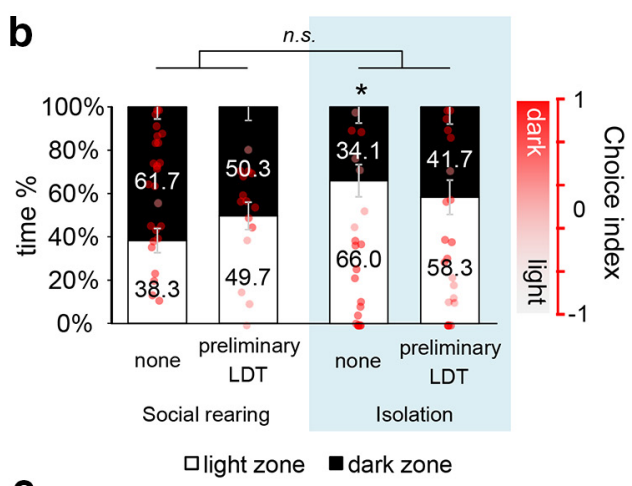

C

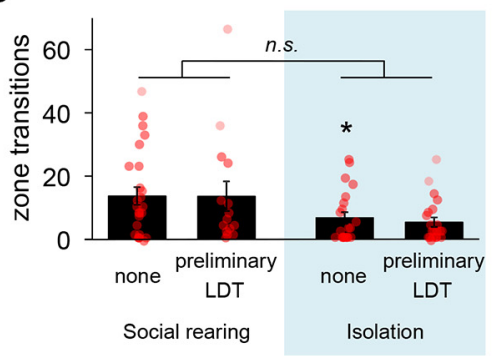

Figure 2. $\quad \boldsymbol{a}$, Protocol of Experiment 2 (top) and schematic drawing of the isolation tanks (bottom). Red timeline represents

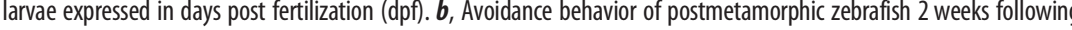

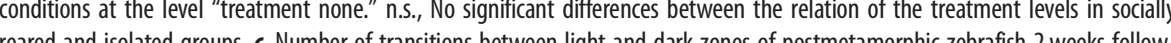
ing a single LDT challenge and/or after 2 weeks of chronic social isolation. LDT: light/dark tank.

respectively. Sampling times were chosen according to the measures of Lau et al. (2011). To analyze environmental reactivity as well in these developmental stages, we repeated the tests on the subsequent days with the same animals and analyzed habituation capacity (see Fig. 1a). Different sets of larvae from the same spawning were tested in each $2 \mathrm{~d}$ trial set. Between the two tests, animals were kept in the isolation tanks described above. Samples sizes were 48 for the $8 \mathrm{dpf}, 24$ for the $14 \mathrm{dpf}$, and 23 for the $28 \mathrm{dpf}$ age groups.

In Experiment 2, we investigated whether chronic absence of social stimuli affects postmetamorphic juvenile behavior and reactivity to acute environmental stimuli (LDT test). We subjected $14 \mathrm{dpf}$ zebrafish to the LDT test and/or 2 weeks of social isolation, then analyzed their avoidance behavior in the LDT test (see Fig. $2 a$ ) at $29 \mathrm{dpf}$. Sample sizes were 25 and 21 (naive and test-experienced) for the socially reared and 15 and 21 (naive and test-experienced) for the isolated groups.

In Experiment 3, we aimed to determine isolation-induced changes in CNS 5-HT content under basal and challenge-induced conditions by conducting HPLC-MS-MS measurements on juveniles before, immediately after, and $1.5 \mathrm{~h}$ after LDT testing. Biological sample sizes were 22, 18 , and 24 (basal, immediate, and $1.5 \mathrm{~h}$ ) for the socially reared and 16 for the isolated groups. Technological samples were pooled from two randomly allocated biological samples.

In Experiment 4, to validate the pharmacological tool to be used for dampening serotonin signaling, we treated socially reared as well as isolated $30 \mathrm{dpf}$ zebrafish with the 5-HT1AR partial agonist buspirone and measured serotonin, 5-HIAA, dopamine, and noradrenaline levels. Biological sample sizes were 25 and 28 (vehicle and buspirone-treated) for the socially reared and 28 for isolated groups. Technological samples were pooled from three randomly allocated biological samples.

In Experiment 5a, to localize isolation-induced changes in 5-HT responses to acute challenges, we subjected juveniles to the LDT test and analyzed 5-HT immunoreactivity throughout the telencephalon $1.5 \mathrm{~h}$ after the stress exposure. To unravel the connection between the observed behavioral and area-specific physiological changes, we aimed to diminish 
5-HT activity by the administration of buspirone, predominantly acting on 5-HT1A autoreceptors (see Fig. 4a). Sample sizes were 31 and 22 (vehicle and buspirone-treated) for the socially reared and 14 and 16 (vehicle and buspirone-treated) for the socially isolated groups.

In Experiment 5b, we aimed to investigate the effects of isolation under basal and stress-induced arousal states and accompanying sensory responsiveness by subjecting juveniles to stationary and moving items projected onto the testing arena. To clarify the role of the serotonin response, we treated the animals with buspirone between basal and stress-induced phases. Sample sizes were 13 and 14 (vehicle and buspirone-treated) in both socially reared and isolated groups as well.

In Experiments $5 c$ and $5 d$, we aimed to investigate the effects of isolation in different contexts by subjecting juveniles to the SPM or the Ushaped social preference tests after a single treatment of buspirone. In addition, to determine whether avoidance in the SPM is visually based, in Experiment 5e we analyzed behavior in brightly lit and dark conditions as well. In Experiment 5c, sample sizes were 28 and 24 (vehicle and buspirone-treated) for the socially reared and 17 and 16 (vehicle and buspirone-treated) for the socially isolated groups; in Experiment 5d, these were 16 and 25 (vehicle and buspirone-treated) for socially reared and 14 for each isolated group, whereas in Experiment 5e, sample size was 20 .

\section{Data analysis}

Data are shown as mean \pm SEM. We performed statistical analyses in R Statistical Environment (R Core Team, 2017). Sample sizes were calculated using the pwr2 package (Pengcheng Lu, Junhao Liu, and Devin Koestler). We defined an effect size of 0.3 in either factors, and a standard power and $\alpha$ of 0.8 and 0.05 , entailing an optimal sample size of 23 . To analyze the interactional effects of rearing conditions, developmental experiences, and pharmacological treatments on behavior, we used linear mixed models (Faraway, 2016) from the lme4 package (Bates, 2015). To analyze these effects on neurotransmitter concentration or immunoreactivity as well, we used linear models. Rearing "social" or rearing "isolated" was set as a reference level of the factor rearing condition, whereas treatment "vehicle" was set as reference level of the factor treatment. To separate variance stemming from time or sequence of experimental trials or location of test platforms, these factors were added as random effects to our models. The statistical output of such models indicates whether the effects of treatments are identical throughout rearing conditions. In this manner, we only showed the results in relation to the reference category; for example, the difference between control and treated isolated groups differs from the corresponding difference between socially reared groups. To analyze within-group differences between repeated measures, we fitted linear mixed models with subject identifiers as random effects. We computed Pearson correlation coefficients ( $r$ ) using the GGally package (Schloerke et al., 2017). We calculated $p$ values from $t$ values of $l m e 4$ using the lmerTest package (Kuznetsova et al., 2017) and rejected $\mathrm{H}_{0}$ if $p$ values were $<0.05$

\section{Results}

\section{Behavioral metamorphosis in zebrafish defines a time window with enhanced environmental reactivity}

Since larval and juvenile zebrafish express a different behavioral repertoire (Lau et al., 2011; Dreosti et al., 2015), it is evident that, in addition to the morphologic metamorphosis, a behavioral reorganization also occurs in this species. We hypothesized that the time period of such transformation may also function as a window of opportunity for individuals to sample current environmental demands and adapt to potential challenges. Such processes require a great amount of environmental reactivity and may represent a sensitive developmental period that may be suitable to model the effects of early-life experiences. In Experiment 1 (Fig. 1), we aimed to determine the time of the switch from the light- to the dark-preferring phenotype, representing a specific aspect of the behavioral metamorphosis (Lau et al., 2011), by subjecting separate sets of zebrafish to the LDT test for $10 \mathrm{~min}$ at
8, 14, and 28 dpf, respectively (Fig. 1a). To characterize environmental reactivity as well in these phases of development, we repeated the experiments the following day $(9,15$, or $29 \mathrm{dpf}$, respectively) with the same animals. While $8 \mathrm{dpf}$ larvae preferred the light over the dark zone, the preference was shifted toward the dark zone in older groups (Fig. 1c), outlining the time onset of the aforementioned phenotypic shift ( $8 \mathrm{dpf} /$ first test $-14 \mathrm{dpf} /$ first test: estimate $=0.892, \mathrm{SEM}=0.119, t=7.516, p=2.48 \mathrm{e}-12 ; 8$ $\mathrm{dpf} /$ first test $-27 \mathrm{dpf} /$ first test: estimate $=0.756, \mathrm{SEM}=0.120$, $t=6.285, p=2.35 \mathrm{e}-09)$. At test repetitions, 9 dpf light-preferring larvae showed increased dark exploration $(8 \mathrm{dpf} /$ first test -8 $\mathrm{dpf} / \mathrm{second}$ test: estimate $=0.256, \quad \mathrm{SEM}=0.098, \quad t=2.630$, $p=0.009), 15$ dpf dark-preferring larvae showed increased light exploration $(14 \mathrm{dpf} /$ first test $-14 \mathrm{dpf} /$ second test: estimate $=$ $-0.513, \mathrm{SEM}=0.136, t=-376, p=0.0002 ; 8 \mathrm{dpf} /$ first-second $\times$ $14 \mathrm{dpf} /$ first-second interaction: estimate $=-0.769, \mathrm{SEM}=0.168$, $t=-4.587, p=8.36 \mathrm{e}-06$ ), while $29 \mathrm{dpf}$ fish expressed no detectable change of behavior compared with the first testing day ( 27 $\mathrm{dpf} /$ first test $-27 \mathrm{dpf} /$ second test: estimate $=0.101, \mathrm{SEM}=0.139$, $t=0.727, p=0.468 ; 8 \mathrm{dpf} /$ first-second $\times 27 \mathrm{dpf} /$ first-second interaction: estimate $=-0.155, \mathrm{SEM}=0.170, t=-0.912$, $p=0.363$ ) (Fig. 1d). It is important to note that $15 \mathrm{dpf}$ larvae showed the most extreme intertest decrease indicated by the slope of change in avoidance (age effect: sum $\mathrm{sq}=9.45 \mathrm{~F}=12.87$, $p=1.24 \mathrm{e}-0.5$; pairwise comparisons, $8 \mathrm{dpf}$ to $14 \mathrm{dpf}$ : estimate $=$ $-0.743, p=0.00002,14 \mathrm{dpf}$ to $27 \mathrm{dpf}$ : estimate $=0.700$, $p=0.0004$ ) (Fig. 1d), and this group had the highest proportion of animals showing habituation, 19 of 24 fish decreased their avoidance (Fig. 1e). Furthermore, we did not detect any differences between 14 and $15 \mathrm{dpf}$ test-naive animals ( $15 \mathrm{dpf}$ repeated $15 \mathrm{dpf}$ naive: estimate $=-0.198, \mathrm{SEM}=0.140, t=-0.417$, $p=0.158$ ), indicating that the observed effects were experience-dependent changes and were not biased by aging. Linear regression showed that, in the 8 and $14 \mathrm{dpf}$ groups, the absolute value of avoidance in the first tests closely predicted the absolute value of slope of change in avoidance between tests $(8 \mathrm{dpf}$ slope $\sim 8 \mathrm{dpf}$ choice index: estimate $=0.426, \mathrm{SEM}=0.111, t=0.825, p=0.00042$; $14 \mathrm{dpf}$ slope $\sim 14 \mathrm{dpf}$ choice index: estimate $=0.524, \mathrm{SEM}=0.262$, $t=1.997, p=0.0583 ; 27 \mathrm{dpf}$ slope $\sim 27 \mathrm{dpf}$ choice index: estimate $=0.195, \quad \mathrm{SEM}=0.286, t=0.681, p=0.503) \quad$ (Fig. 1f $)$. Extreme first-trial avoidance was associated with stronger intertest changes in behavior. In summary, the first 2 weeks of development mark a specific aspect of behavioral metamorphosis and a period of enhanced reactivity that peaks at the second sampling event.

\section{Social isolation in the reactive period disrupts later behavioral responsiveness of zebrafish}

Given such increased environmental reactivity around the second week of development, in Experiment 2, we assessed the effects of different experiences gained in this time window on the postmetamorphic behavioral phenotype. We subjected zebrafish larvae to acute novelty stress (LDT testing) at 14 and/or chronic environmental isolation from 14 to $28 \mathrm{dpf}$ and then analyzed their avoidance behavior in the LDT test at the juvenile stage (Fig. 2a). The chronic environmental deprivation started at the most plastic sampling point where behavioral development has occurred and ended after the morphologic metamorphosis; hence, we excluded the chance of detecting a possible conservation event of the immature avoidance phenotype. Environmental isolation, regardless of the onset of preliminary LDT testing, decreased avoidance behavior (soc/noLDT - iso/noLDT: estimate $=-0.530$, SEM $=0.186, t=-2.853, p=0.006$; soc $/$ noLDT soc/LDT: estimate $=-0.208, \mathrm{SEM}=0.207, t=-1.005, p=0.318$; 
soc/noLDT-LDT $\times$ iso/noLDT-LDT interaction: estimate $=0.338, \mathrm{SEM}=0.283$, $t=1.193, p=0.237$ ) (Fig. 2b), the number of transitions between zones (soc/noLDT - iso/noLDT: estimate $=-9.773$, SEM $=$ $3.615, t=-2.704, p=0.008$; soc/noLDT soc/LDT: estimate $=-0.286, \mathrm{SEM}=3.775$, $t=-0.076, p=0.940 ;$ soc/noLDT-LDT $\times$ iso/noLDT-LDT interaction: estimate $=$ 1.411, $\mathrm{SEM}=5.361, t=0.263, p=0.793)$ (Fig. 2c) and mean velocity (data not shown), indicating an overall impairment in responsivity.

\section{Social isolation differentially affects central 5-HT levels of zebrafish under resting and novel conditions}

The decreased response capacity observed led us to investigate the role of 5-HT in isolation-induced effects, since serotonergic signaling is linked to behavioral constraint and environmental responsiveness as well (for review, see Brodie and Shore, 1957; Depue and Spoont, 1986; Soubrié, 1986; Spoont, 1992; Lucki, 1998). To characterize the dynamics of potential 5-HT level changes in our isolation model, we conducted HPLC-MS-MS measurements on whole-brain samples of separate sets of zebrafish under resting conditions, as well as immediately and $1.5 \mathrm{~h}$ after the postmetamorphic LDT test, respectively (Fig. $3 a$ ). Socially reared fish showed a rapid decrease in 5 -HT content in response to LDT testing, changes that were detectable for at least as long as $1.5 \mathrm{~h}$ (soc/basal - soc/LDT: estimate $=-56.810, \mathrm{SEM}=13.448, t=$ $-4.224, p=0.0001$; soc/basal - soc $/ 1.5 \mathrm{~h}$ : estimate $=-75.748$, $\mathrm{SEM}=12.489, t=-6.065, p=1.73 \mathrm{e}-07)$. In contrast, isolated fish showed decreased baseline levels compared with socially reared conspecifics (soc/basal - iso/basal: estimate $=-50.512, \mathrm{SEM}=$ 13.903, $t=-3.633, p=0.0007)$ and a markedly enhanced, transient 5-HT response to LDT testing (iso/basal - iso/LDT: estimate $=45.029, \mathrm{SEM}=14.960, t=3.010, p=0.004$, iso $/$ basal iso $/ 1.5 \mathrm{~h}$ : estimate $=-5.132, \mathrm{SEM}=14.960, t=0.343, p=0.733$ ) (Fig. $3 b$ ). Such differentially directed 5-HT responses to the novelty-challenge were accompanied by a similar decrease in light avoidance that we measured in Experiment 2. Interestingly, minute-by-minute analysis revealed that the principal component of such low responsivity was the delayed emergence of avoidance activity (soc/timebin $\times$ iso/timebin interaction: estimate $=$ -0.029 , SEM $=0.013, t=-2.181, p=0.030$ ), whereas decreased locomotion was stable throughout the test (soc/timebin $\times$ iso/ timebin interaction: estimate $=-0.056, \mathrm{SEM}=0.112, t=-0.501$, $p=0.016)($ Fig. $3 c, d)$.

Isolation-induced changes in serotonin and 5-HIAA, but not in dopamine or noradrenaline levels, is prevented by 5HT1AR partial agonist buspirone

In Experiment 4, we aimed to validate a pharmacological tool suitable for specific blockade of serotonergic signaling. We hypothesized that the clinical anxiolytic agent buspirone might possibly be able to dampen central 5-HT level since (1) it predominantly acts on 5-HT1A receptors (Loane and Politis, 2012), (2) zebrafish express these mainly as autoreceptors of the raphe nuclei (Norton et al., 2008), which are (3) the only serotonergic cell clusters that project to the teleostean telencephalon
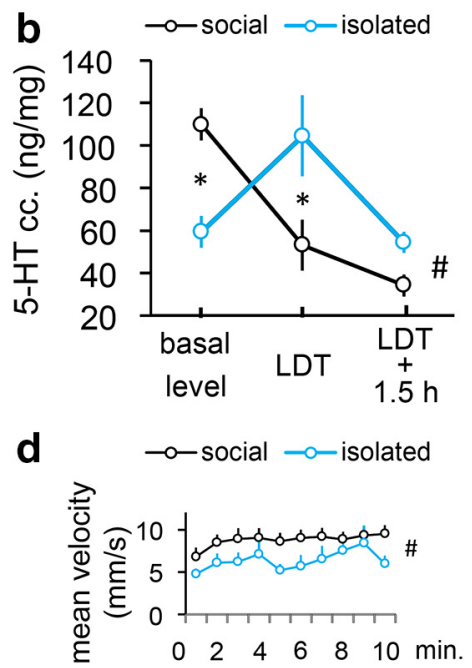

$\begin{array}{llllll}0 & 2 & 4 & 6 & 8 & 10\end{array} \mathrm{~min}$.

Figure 3. $a$, Protocol of Experiment 3. Red timeline represents the age of larvae expressed in days post fertilization (dpf). -brain 5-HT concentration of postmetamorphic zebrafish in resting conditions, and immediately and $1.5 \mathrm{~h}$ after LDT

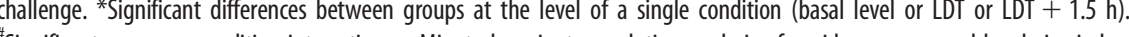
Significant group $\times$ condition interaction. c, Minute-by-minute resolution analysis of avoidance measured by choice index. d, Velocity. "Significant group $\times$ condition interaction. LDT: light/dark tank.

(Maximino et al., 2013a), (4) the area that orchestrates dark avoidance behavior (Lau et al., 2011). Furthermore, (5) buspirone binds to 5-HT1A receptors of zebrafish with a 4 times greater affinity than that of rats and an 8 times greater affinity than that of humans (Barba-Escobedo and Gould, 2012). However, buspirone also modifies dopamine and noradrenaline levels in mammals (Gobert et al. 1997, 1999; Lechin et al., 1998); hence, it was important to assess these neurotransmitters as well. In addition, it is reported several times that buspirone decreases serotonin turnover (Peroutka, 1988; Shireen and Haleem, 2005), an effect that is potentially able to compensate its negative impact on the bioavailability of serotonin. For these reasons, we treated socially reared and isolated juvenile zebrafish with buspirone ( $50 \mathrm{mg} / \mathrm{L}$ ) before LDT testing and measured 5-HT, 5-HIAA, dopamine, and noradrenaline from whole-brain samples, using HPLC-MS-MS (Fig. 4). We reproduced challenge-induced enhancement of 5-HT concentration at the level of the whole brain (soc/control - iso/control: estimate $=5.990, \mathrm{SEM}=2.923$, $t=-2.050, p=0.049$ ), which was prevented by a single preliminary buspirone treatment (iso/control - iso/busp: estimate = $-5.781, \mathrm{SEM}=2.836, t=-2.039, p=0.050)$. We also measured an isolation-induced increase in 5-HIAA level (soc/control - iso/ control: estimate $=2.283, \mathrm{SEM}=1.064, t=2.147, p=0.040$ ), which was prevented by buspirone (iso/control - iso/busp: estimate $=-2.605, \mathrm{SEM}=1.032, t=-2.525, p=0.017)$, a similar pattern to that of what we have shown in 5-HT, indicating no change in serotonin turnover in response to the investigated factors. Interestingly, we found an isolation-induced enhancement in dopamine as well (soc/control - iso/control: estimate $=69.991$, $\mathrm{SEM}=29.524, t=2.371, p=0.024)$, that was insensitive to buspirone treatment (iso/control - iso/busp: estimate $=-34.97$, $\mathrm{SEM}=28.64, t=-1.221, p=0.231$ ), whereas we did not find any change in noradrenaline in response to the assessed conditions (soc/control - iso/control: estimate $=780.9, \mathrm{SEM}=587.4, t=$ 1.129, $p=0.193$; iso/control - iso/busp: estimate $=-1061$, $\mathrm{SEM}=569.9, t=1.852, p=0.072$ ). In summary, we found that isolation-induced change in central serotonin, but not dopamine or noradrenaline levels, is pharmacologically prevented by the 
a
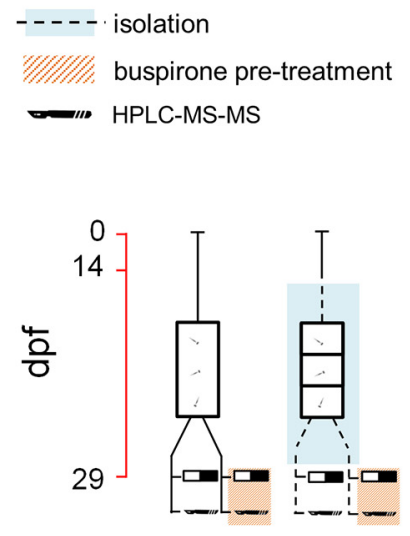

d

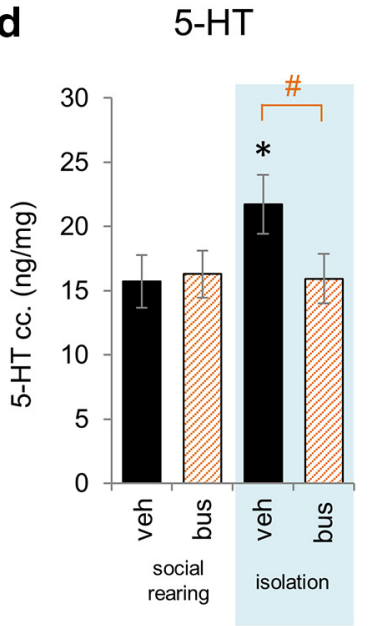

b

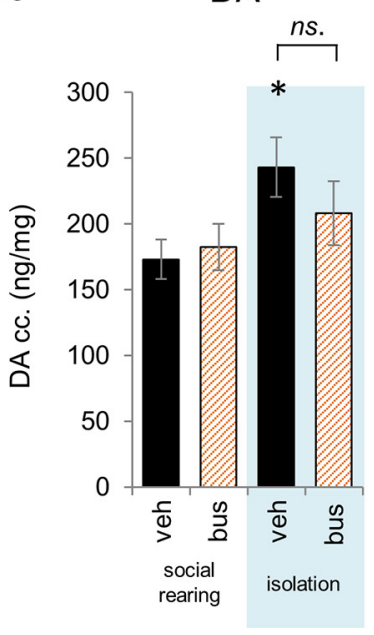

e

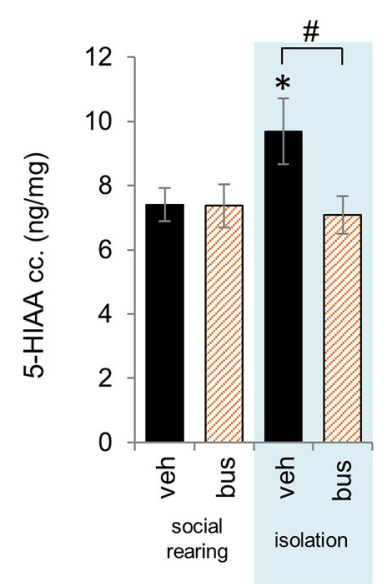

C

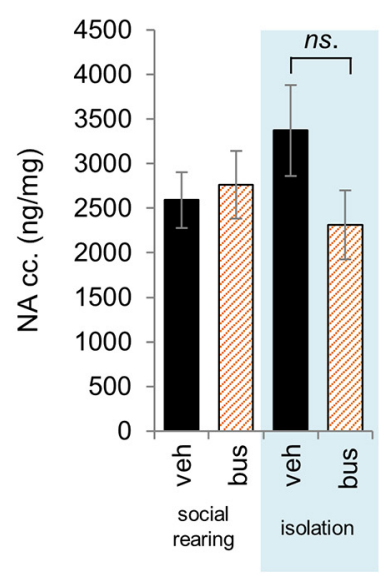

f

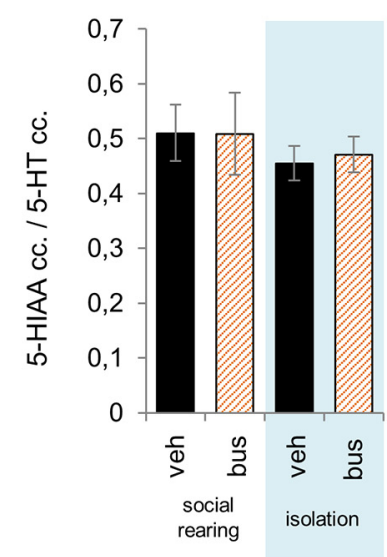

Figure 4. a, Protocol of Experiment 4a. Whole-brain concentration of (b) dopamine, (c) noradrenaline, (d) 5-HT, (e) 5-HIAA, and ( $\boldsymbol{f})$ the ratio of 5-HIAA and 5-HT concentrations as an indicator of 5 -HT turnover of postmetamorphic zebrafish 2 weeks following chronic social isolation and/or acute buspirone treatment. *Significant difference from socially reared vehicle-treated group. Black \# indicates significant difference from socially isolated vehicle-treated group. Orange \# indicates marginal significance $(p=0.050)$. ns., No significant. veh: vehicle, bus: buspirone.

acute administration of buspirone. In addition, isolation-, as well as buspirone-induced changes are potentially independent from serotonin turnover.

Social isolation-induced 5-HT changes are specific to the dorsal pallium and, along with behavioral deficits, can be prevented by blockade of 5-HT signaling

In Experiment 5a, we assessed the functional connectivity between isolation-induced behavioral and physiological effects. We administered buspirone before the postmetamorphic LDT test and used immunohistochemical labeling for 5-HT in several regions of the telencephalon as well (Fig. 4c). Isolated fish, similarly to those in Experiments 2 and 3, showed decreased light avoidance behavior (soc/control - iso/control: estimate = $-0.335, \mathrm{SEM}=0.155, t=-2.159, p=0.034$ ). This effect was preventable by an acute buspirone treatment (iso/control - iso/ busp: estimate $=0.685, \mathrm{SEM}=0.179, t=3.815, p=0.0003$ ) with a dose that had no effect on socially reared conspecifics (soc/control - soc/busp: estimate $=0.021, \mathrm{SEM}=0.142, t=0.148, p=$ 0.883 ) (Fig. 5b). Immunohistochemical labeling revealed higher 5-HT immunoreactivity in isolated fish compared with controls in the lateral forebrain bundle (LFB soc/control - iso/control: estimate $=3.004, \mathrm{SEM}=1.131, t=2.656, p=0.012)$ and in the caudal (DPc soc/control - iso/control: estimate $=2.758, \mathrm{SEM}=$ $1.011, t=2.729, p=0.010)$, but not in any rostral part of the pallium (DPr soc/control - iso/control: estimate $=-0.611, \mathrm{SEM}=$ 0.726, $t=-0.841, p=0.410$; $\mathrm{VPr}$ soc/control - iso/control: estimate $=-0.314, \mathrm{SEM}=0.373, t=-0.841, p=0.409)$. These differences were abolished by the acute administration of buspirone (LFB iso/control - iso/busp: estimate $=-3.004, \mathrm{SEM}=1.131$, $t=-2.656, p=0.012 ; \mathrm{DPc}$ iso/control - iso/busp: estimate $=$ $-2.320, \mathrm{SEM}=1.078, t=-2.153, p=0.039$ ) (Fig. $5 d, e$ ). Together, our results display that, with a single 5-HT1AR agonist treatment, we were able to restore the normal novelty-induced avoidance phenotype. In addition, we found 5-HT level differences in fibers that enter the forebrain and target the caudal recess of the telencephalon where cell masses of the dorsomedial and dorsolateral areas, homolog structures of the mammalian amygdala and hippocampus, emerge separately (Wulliman et al., 2012). Such differences were preventable by acute buspirone treatment. In summary, we detected functional connections between forebrain-localized 5-HT response and inadequate avoidance behavior. 
a
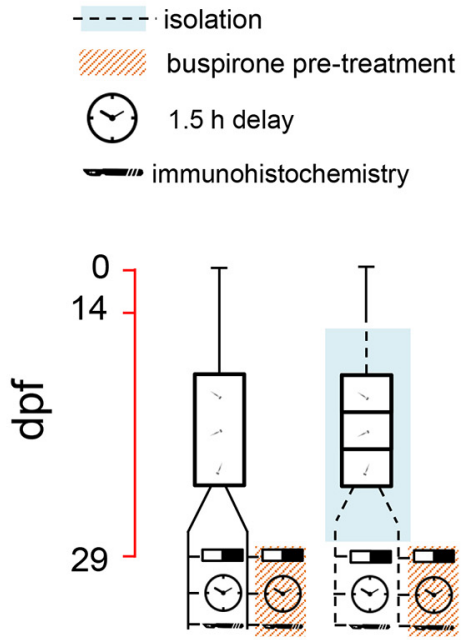

b

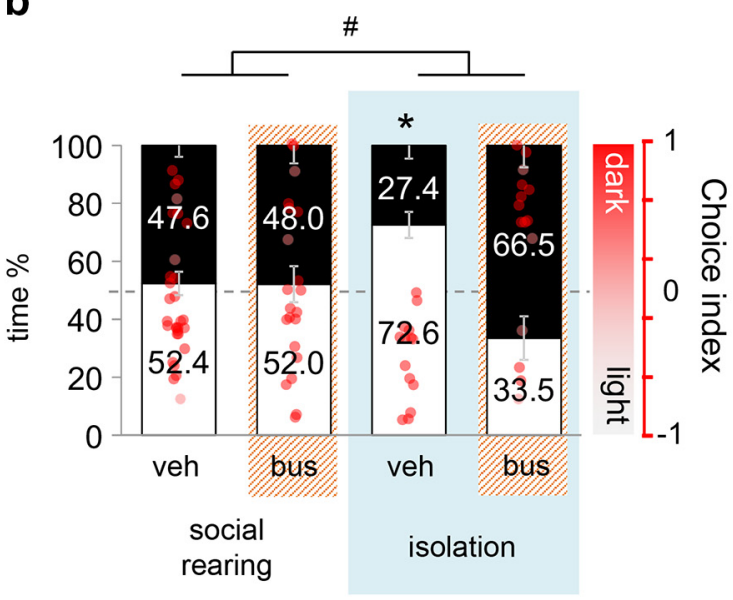

$\square$ light zone $\square$ dark zone

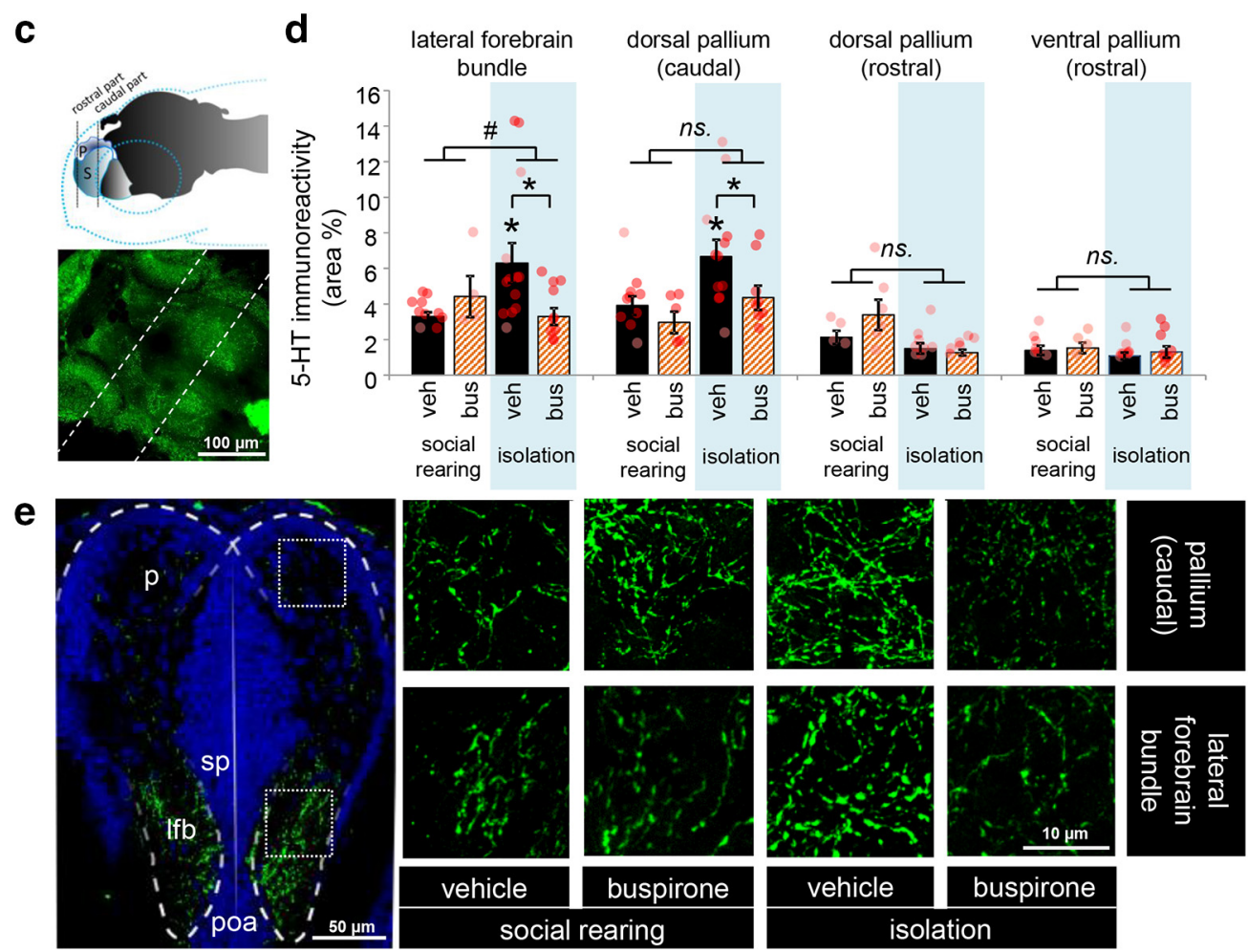

Figure 5. $\boldsymbol{a}$, Protocol of Experiment 5a. $\boldsymbol{b}$, Avoidance behavior of postmetamorphic zebrafish 2 weeks following chronic social isolation and/or after acute buspirone treatment. Left axis indicates the percentage of time. Right axis indicates choice index in which 1 indicates $100 \%$ time in the dark zone, whereas -1 represents $100 \%$ time in the light zone. Reference levels of the linear mixed model were "social rearing" and "treatment vehicle." *Significant differences between rearing conditions at the level of treatment vehicle. "Significant differences between the relation of the treatment levels in socially reared and isolated groups. c, Schematic drawing (top) and confocal microphotographs (maximum intensity projection) (bottom) of the investigated areas. The image was taken from a whole-brain preparatum of a zebrafish. Dashed lines indicate the approximate anteroposterior position of the investigated areas. $\boldsymbol{d}$, Quantification of 5 -HT immunoreactivity in the analyzed brain regions. *Significant differences between rearing conditions at the level of treatment vehicle. "Significant differences between the relation of the treatment levels in socially reared and isolated groups. $\boldsymbol{e}$, Representative confocal microphotographs (maximum intensity projection) of sites with significant differences in immunoreactivity. ns., No significant. veh: vehicle, bus: buspirone.

Social isolation shifts response capacity between novel and resting conditions and such effect is inverted by the blockade of 5-HT signaling

Because of the highly different levels of 5-HT in resting and novel environment, including changes in limbic structures, and ineffective challenge responding of isolated fish, in Experiment
$5 \mathrm{~b}$, we assessed possible isolation-induced impairment in stressinduced arousal state. Alertness in mammals is associated with activation of the amygdala (Gläscher and Adolphs, 2003; Harmer et al., 2006) and modulated by 5-HT signaling (Spoont, 1992); hence, we speculated that an inadequate emergence of such condition may drive the isolation-induced behavioral impairment of 
a<smiles>OCCOCCOCc1ccccc1OCCl</smiles>

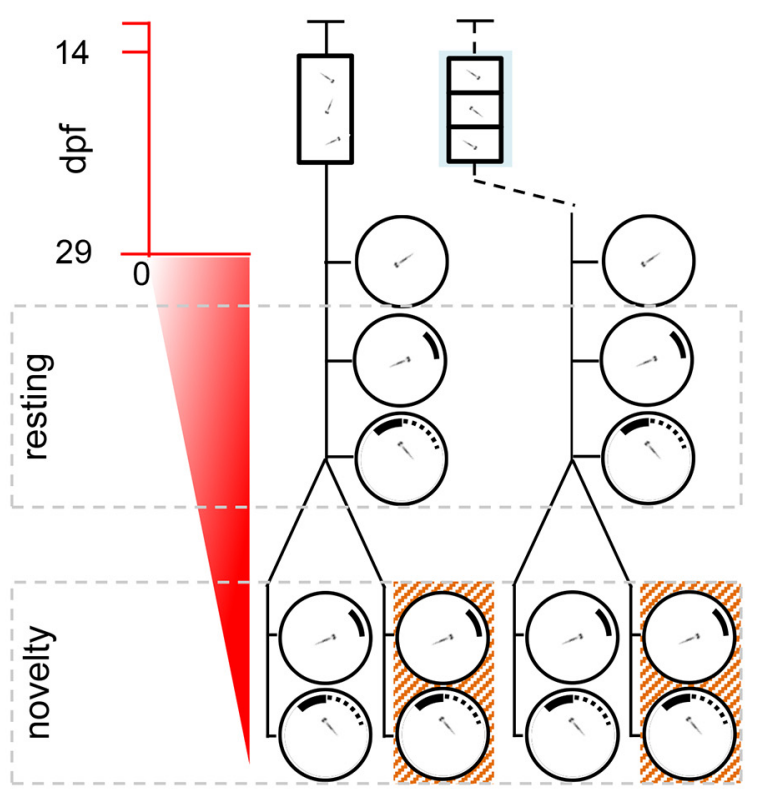

$c \%$

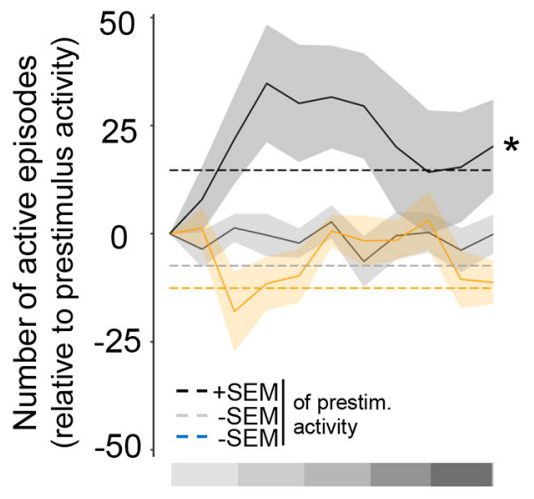

Socially reared

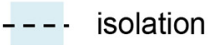

buspirone

pre-treatment

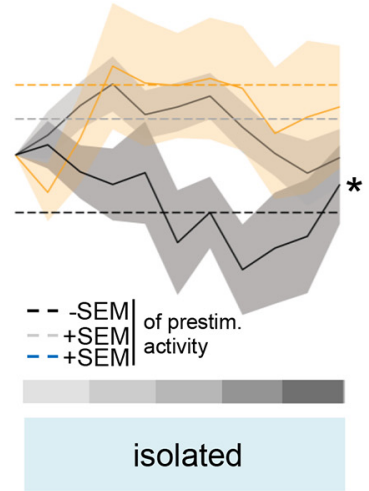

b
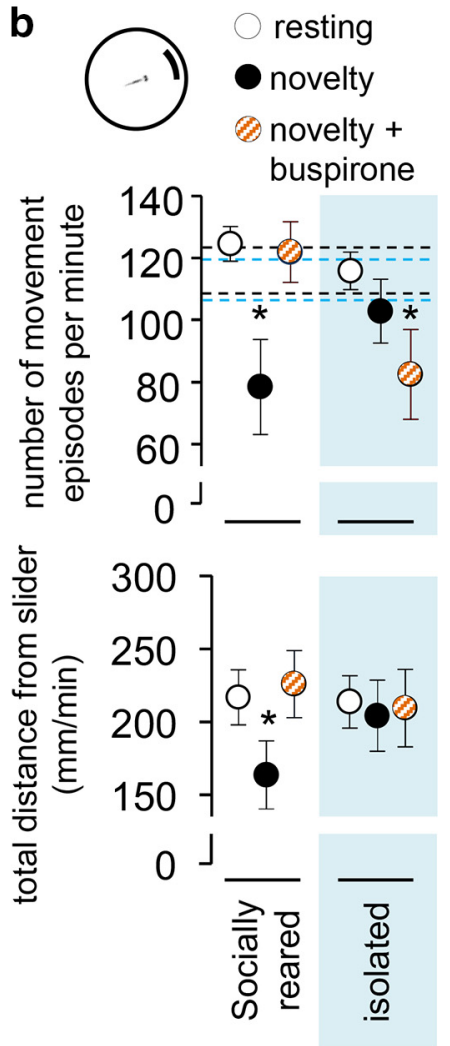

d
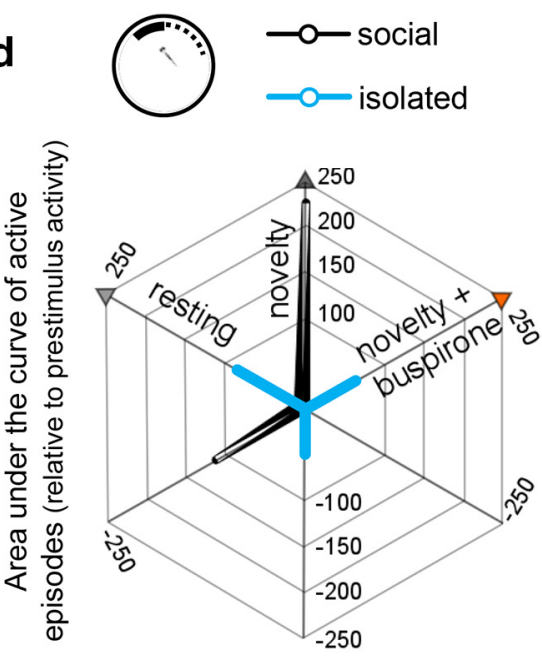

Figure 6. $\boldsymbol{a}$, Protocol of Experiment $5 \mathrm{~b}$. Red timeline represents the age of larvae expressed in days post fertilization (dpf). $\boldsymbol{b}$, Locomotor response to (top) and the total distance from (bottom) the projected stationary object in resting and novel conditions and in response to prior buspirone treatment. Reference levels of the linear mixed model were "social rearing" and "condition resting." *Significant differences between experimental conditions (resting, novelty, novelty + buspirone) at the level of each rearing conditions. Dashed lines indicate \pm SEM of activity during the habituation period. c, Minute-by-minute resolution analysis of locomotor response to the moving object in resting and novel conditions and in response to prior buspirone treatment. The number of active swimming episodes are presented relative to previous (prestim) activity of the individuals. Band represents \pm SEM. Gray gradient scale represents the dynamics of speed increase of the moving object. Dashed lines indicate SEM of the prestimulus activity. *Significant differences from resting condition. $\boldsymbol{d}$, Arousal profiles of socially reared and isolated animals presented as locomotor responses to the moving object under different conditions (resting, novelty, novelty + buspirone). 3D starplot represents the three different conditions. Locomotor response is presented as the area under the curve of active episodes relative to the previous activity of the individuals.

zebrafish. In order to assess this issue, a stationary black slider was projected on the bottom of testing compartments for $5 \mathrm{~min}$, which subsequently started to move in circles with increasing speed (Fig. 6a). We sampled behavioral responses in resting conditions, after $20 \mathrm{~min}$ of habituation, as well as in novel environment, immediately after the transfer to the apparatus. Normally, an animal in novelty-induced alert state reacts more rapidly to movement or reacts to a yet slower moving object (Yokogawa et al., 2012) compared with habituated resting individuals. Socially reared animals, in novel but not in familiar context, showed a decrease in the number of swimming episodes (swimming episodes, soc/resting - soc/novely: estimate $=-44.972$, SEM $=$ 

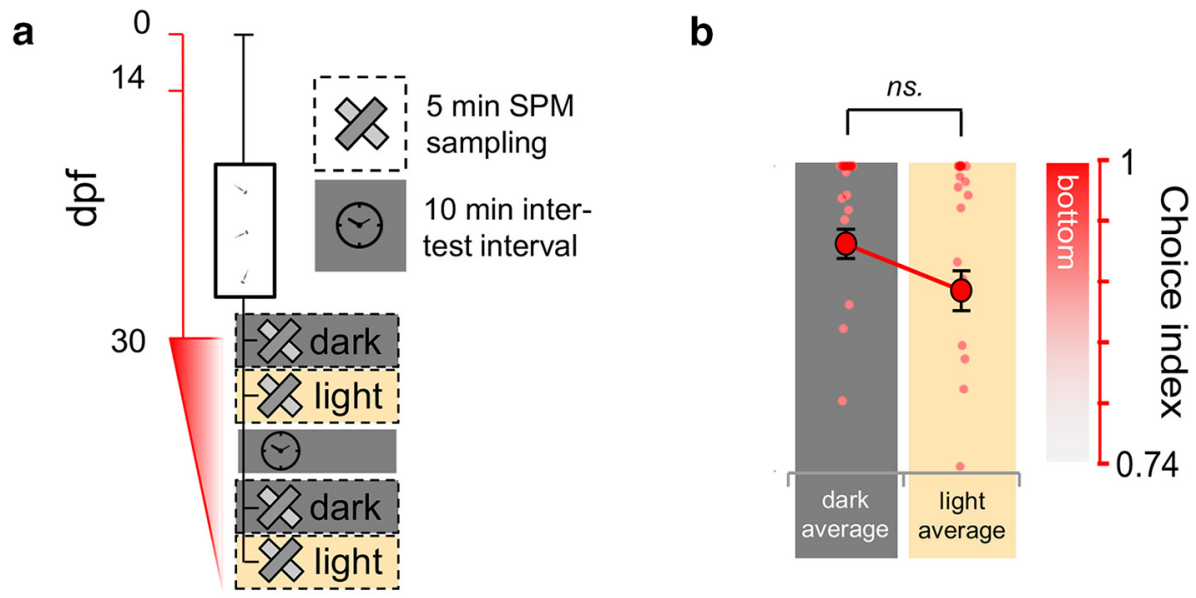
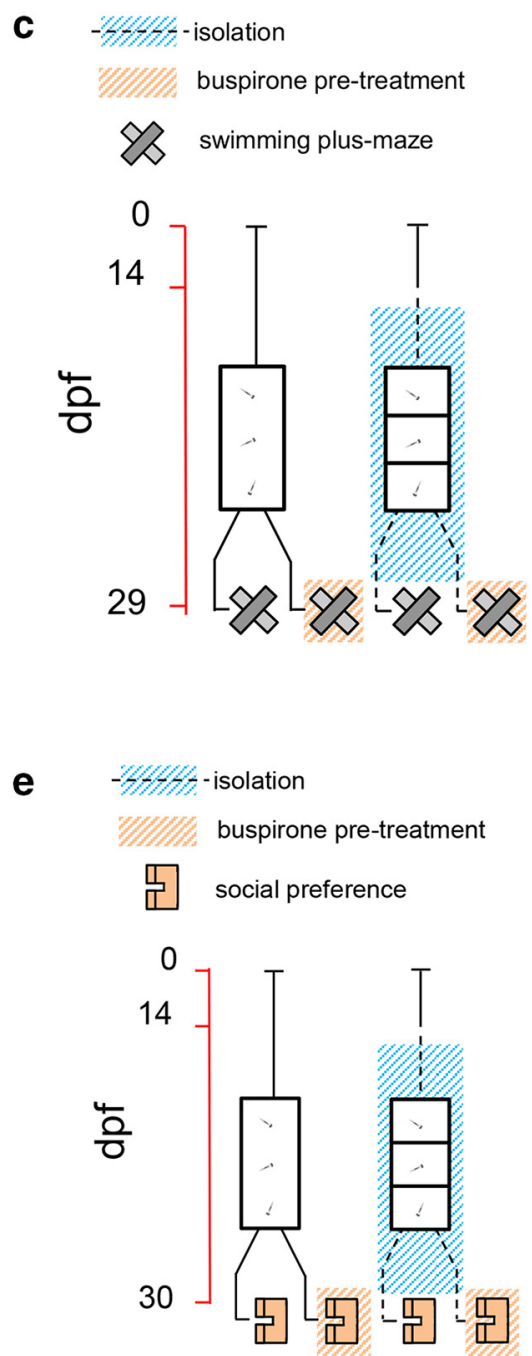

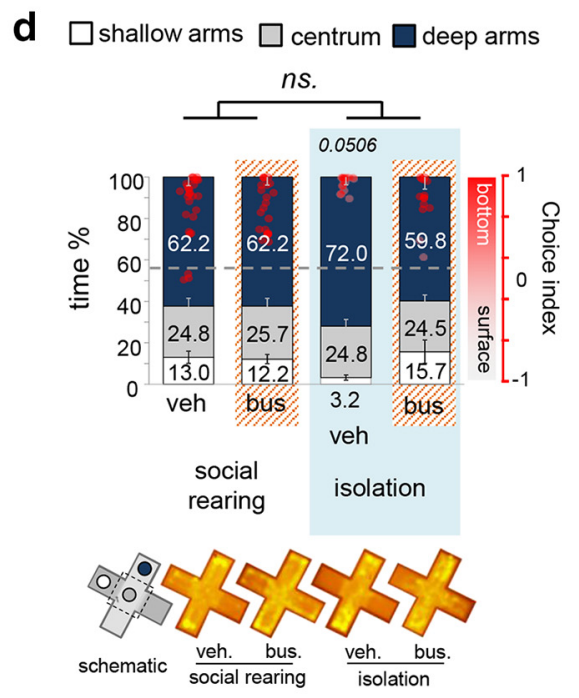

f $\square$ social chamber $\square$ empty chamber

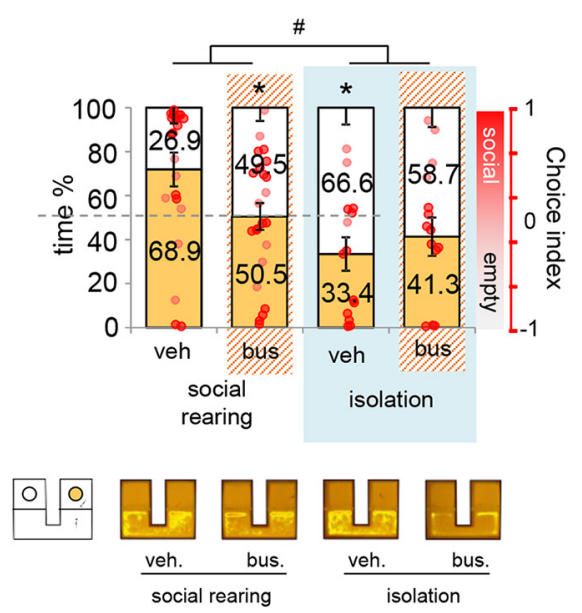

Figure 7. $\boldsymbol{a}$, Protocol of Experiment 5e. Red timeline represents the age of larvae expressed in days post fertilization (dpf). $\boldsymbol{b}$, Average surface avoidance behavior of socially reared untreated juvenile zebrafish in dark and lit environment. Right axis and red dots represent choice index in which 1 indicates $100 \%$ time in the deep zone, whereas -1 represents $100 \%$ time in the shallow zone. c, Protocol of Experiment 5c. $\boldsymbol{d}$, Surface avoidance behavior of postmetamorphic zebrafish 2 weeks following chronic social isolation and/or acute buspirone treatment (top) and their representative heatmaps (bottom). Left axis indicates the percentage of time. Right axis and red dots represent choice. The reference levels of the linear mixed model were "social rearing" and "treatment vehicle." $\boldsymbol{e}$, Protocol of Experiment 5 d. $\boldsymbol{f}$, Social preference of postmetamorphic zebrafish 2 weeks after chronic social isolation and/or after buspirone treatment (top) and their representative heatmaps (bottom). Left axis indicates the percentage of time. Right axis and red dots represent choice index in which 1 indicates $100 \%$ time in the social chamber, whereas - 1 represents $100 \%$ time in the empty chamber. The reference levels of the linear mixed model were "social rearing" and "treatment vehicle." *Significant differences from socially reared vehicle treated animals. "Significant differences between the relation of the treatment levels in socially reared and isolated groups. ns., No significant. veh: vehicle, bus: buspirone. 
11.602, $t=-3.876, p=0.0002)$ and in the distance from the stationary slider (distance from item, soc/resting - soc/novely: estimate $=-55.982, \mathrm{SEM}=27.018, t=-2.072, p=0.042)$ in response to its appearance, whereas buspirone prevented both of these effects (swimming episodes, soc/resting - soc/busp: estimate $=-4.074, \mathrm{SEM}=12.377, t=-0.329, p=0.743$; distance from item, soc/resting - soc/busp: estimate $=-10.613, \mathrm{SEM}=$ 28.864, $t=-0.368, p=0.714$ ) (Fig. 6b). Interestingly, isolated fish only showed such stimuli-induced decreased locomotion in response to buspirone treatment (swimming episodes, iso/resting - iso/novelty: estimate $=-12.591, \mathrm{SEM}=12.223, t=-1.131, p=$ 0.307 ; iso/resting - iso/busp: estimate $=-33.707, \mathrm{SEM}=11.157$, $t=-3.021, p=0.004)$. In response to the movement stimulus, socially reared animals increased the frequency of their swimming episodes in novel but not in resting conditions (soc/resting - soc/novelty: estimate $=17.476, \mathrm{SEM}=8.341, t=2.095, p=$ 0.037), whereas buspirone abolished this reaction (soc/resting soc/busp: estimate $=2.498, \quad$ SEM $=8.531, t=0.293, p=0.770$ ) (Fig. 6c). In contrast, isolated fish showed higher response frequency in resting, and a delayed, oppositely directed response in stress-induced conditions (iso/resting - iso/novelty: estimate $=$ 21.406, $\mathrm{SEM}=8.773, t=2.440, p=0.015)$. Buspirone pretreatment of isolated fish triggered a response similar in shape that of socially reared stressed or isolated habituated animals (iso/resting - iso/busp: estimate $=-4.788, \mathrm{SEM}=8.447, t=-0.567$, $p=0.571)$. In summary, we detected an isolation-induced lack of typical stress-induced, arousal-associated activity in novel, and an exacerbated activity in resting conditions, whereas buspirone evoked opposite direction effects compared with socially reared animals.

\section{Social isolation-induced decrease in responsiveness is specific to novelty-based visually driven challenges}

Given such isolation-induced low responsivity, potentially driven by an inability to form stress-associated arousal, next we investigated whether the effects are challenge-specific. In order to assess this issue, in Experiments $5 c$ and $5 d$, we analyzed isolationinduced changes in response to different environmental challenges, such as the SPM (Varga et al., 2018) and the U-shaped social preference tests (Dreosti et al., 2015). Similarly to the LDT challenge, both tests trigger approach-avoidance conflict in the animal, however, presenting different type of stimuli (e.g., nonvisual nonmeaningful [novelty] and visual meaningful [social] challenges). To assess the importance of visual cues in the SPM, in Experiment 5e, we also conducted SPM tests in lit and dark environment as well, using healthy juveniles (Fig. 7a), and found no difference in shallow arm activity (light average - dark average: $t=-1.386, \mathrm{df}=19, p=0.182$ ) (Fig. $7 b$ ). In Experiments $5 c$ and $5 \mathrm{~d}$, we analyzed the potential role of 5-HT signaling as well, by the administration of buspirone before the tests (Fig. 7c). Interestingly, isolated fish showed increased avoidance compared with socially reared controls in both tests (SPM, soc/control iso/control: estimate $=-0.237, \mathrm{SEM}=0.120, t=-1.984, p=$ 0.051 ; SP, soc/control - iso/control: estimate $=-0.790, \mathrm{SEM}=$ $0.208, t=-3.802, p=0.0003)$; however, buspirone completely (SPM, iso/control - iso/busp: estimate $=-0.313, \mathrm{SEM}=0.135$, $t=-2.317, p=0.023$ ) (Fig. $7 d$ ) or partially (SP, soc/control - iso/ busp: estimate $=-0.522, \mathrm{SEM}=0.209, t=-2.498, p=0.015)$ (Fig. $7 f$ ) prevented such effects. In the case of the social preference test, buspirone abolished social activity regardless of its direction (e.g., the preference in case of socially reared and the aversion in case of isolated animals: soc/control-busp $\times$ iso/ control-busp interaction: $\quad$ estimate $=0.703, \quad$ SEM $=0.292$, $t=2.404, p=0.019)$. These results indicate that decreased responsiveness of isolated animals is specific to visual nonmeaningful, novel stimuli (e.g., the light/dark or the sensory-motor reaction tests).

\section{Discussion}

We have characterized a period during zebrafish development that is highly reactive to the environment in terms of the ability to change behavioral responses to a repeated challenge. Absence of social stimuli during this period promoted an atypical challenge-coping phenotype, characterized by exacerbated alertness under resting conditions and inability to show novelty-induced arousal and avoidance behavior. In line with these behavioral impairments, isolated fish expressed lower baseline, and same magnitude, but oppositely directed serotonin-level changes in response to novelty compared with socially reared subjects. This effect was localized in the caudal excess of the dorsal pallium, a brain area responsible for adequate stress-induced aversion. 5HT1AR agonist treatment prevented isolation-induced central serotonin response without affecting catecholamine levels and rescued physiological challenge-induced behaviors as well, indicating a functional connection between these processes. The effects were specific to visual challenges in a novel context, since they were undetectable at nonvisual novelty, and visual social challenges.

In our first experiments, we showed that a switch from dark to light avoidance, a specific aspect of the behavioral metamorphosis, occurs between the first and second week of development in zebrafish. Furthermore, we showed that this process overlaps with the ability to decrease avoidance in response to repeated challenge, suggesting that this switch is accompanied by enhanced behavioral plasticity and, thus, may serve as a tool to model early life experience-dependent phenomena. The light/ dark choice reversal was first observed by Lau et al. (2011), who estimated its occurrence later, between the second and third week of development. This discrepancy indicates a potential experience-dependent flexibility in the onset of such process, while such variability in the onset supports our hypothesis that the first month of ontogenesis possibly represents a highly plastic, reactive developmental phase. Decreased avoidance at repeated testing supports this idea as well, as it may be driven by habituation, a neuroplasticity-based process (Thompson and Spencer, 1966).

In our next experiments, we showed that the absence of social stimuli during this sensitive period exerted robust effects on behavior, including decreased avoidance and locomotion in the LDT test. Since this test, used here to mimic an acute environmental challenge, is a pharmacologically (Gould, 2011; Maximino et al., 2011; Chen et al., 2015) physiologically (Chen et al., 2015), and behaviorally (Blaser and Peñalosa, 2011) validated tool for measuring anxiety-associated behavior of zebrafish, such decreased avoidance might be interpreted as social isolationinduced anxiolysis. This theory, however, is unlikely, as (1) application of the anxiolytic agent buspirone prevented the decrease; thus, if the "anxiolysis hypothesis" is accepted, it acted as an anxiogenic drug, contradicting its widely accepted effect on behavior; and (2) phenomenological correlates of other anxiety-like domains were enhanced in response to social isolation. According to rodent and zebrafish literature, it is rare, but not unique (Parker et al., 2012; Shams et al., 2015), that such a chronic environmental perturbation as social isolation promotes a low avoidance phenotype (for review, see Hall, 1998; Fone and 
Porkess, 2008; Lukkes et al., 2009a); in particular, several authors discussed social isolation of zebrafish as beneficial, since it consistently decreased stress-induced cortisol response and in some cases anxiety as well (Parker et al., 2012; Giacomini et al., 2015; Shams et al., 2015; Forsatkar et al., 2017). Despite the importance of these results, we suggest a more careful interpretation of the above data. Clinical and preclinical data suggest that an inadequate cortisol response may drive or at least coincide with abnormal behavior, regardless of the direction in which it moves out of the physiological range (Vanyukov et al., 1993; van Goozen et al., 1998; McBurnett et al., 2000; Haller et al., 2001; Toth et al., 2011; Gutiérrez et al., 2019). Furthermore, the corticosteroid response is not necessarily in a positive or in any relation with the expression of anxiety-like behavior (Mikics et al., 2005; Aliczki et al., 2013). On the other hand, isolation-induced low avoidance has also been interpreted as reduced proactive coping in rodents. Isolated rats consistently showed adversity-dependent delay in exploration, indicating a stress-related incapability of challenge responsiveness (Arakawa, 2005, 2007). Also, zebrafish individuals that show delayed emergence from a dark to a brightly lit arena, a phenotype resembling the one observed in our study, showed lower locomotion in general and lower challenge-induced but similar baseline HPA axis activity (Tudorache et al., 2015). This link is further supported by Xin et al. (2020) who found that early-life physiological stress, such as the administration of the synthetic glucocorticoid prednisolone, induces later stress-axis hypofunction, decreased ability to react light-dark transitions, and an overall decrease in exploration. According to these data, one can hypothesize that inefficient physiological stress-response and low avoidance are linked phenomena and maintain inadequate challenge-responding. This background may account for low avoidance in adult and decreased locomotion in $30 \mathrm{dpf}$ juvenile zebrafish after social isolation as well, observed by Shams et al. (2015, 2017a). Our findings on isolation-induced low, delayed avoidance and inadequate arousal in response to a challenge are consistent with the above results and suggest a new interpretation of those. We suggest that isolated fish shift stress-associated alertness to baseline conditions and in this manner are unable to develop adequate internal states and accompanying behavioral responsiveness in either of the contexts. This also means that lack of stimuli during the characterized, highly plastic developmental period has a crucial role in shaping the postmetamorphic behavioral stress responsiveness.

Given the low responding behavioral phenotype, we assessed isolation-induced effects on 5-HT signaling since it is highly sensitive to early life adversity and is known as a prominent modulator of mood, as well as general or specific arousal states (Soubrié, 1986; Spoont, 1992; Lucki, 1998). We found that isolated fish show decreased baseline 5-HT tone and, contrarily to socially reared conspecifics, a rapid gradual 5-HT increase in response to the LDT challenge. This difference was nonsignificant $1.5 \mathrm{~h}$ later on a whole-brain level; however, it was still detectable in fibers projecting to pallial areas (e.g., the lateral forebrain bundle and the caudal recess of the dorsal pallium). Furthermore, 5-HT1AR agonist treatment prevented differences in 5-HT immunoreactivity at the level of the forebrain, and changes in 5-HT content at the level of the whole brain, without any effect on catecholamine concentrations. Our findings regarding brain 5-HT levels are in contradiction with previous reports on zebrafish; however, this discrepancy might be explained by the facts that, in these papers, (1) only extracellular 5-HT content was measured and (2) adult animals were used (Maximino et al., 2013b), or (3) social isolation was conducted in adult fish or in larvae from the age of 0 dpf (Shams et al., 2015, 2017a,b). The latter case, together with rodent data, supports that the period during which isolation occurs is fundamental regarding later consequences, highlighting the importance of critical and sensitive developmental periods (Einon and Morgan, 1977; Hall, 1998; Fone and Porkess, 2008; Lukkes et al., 2009a). We suggest that the environmental perturbation we applied in a period characterized by enhanced adaptive capacity exerts neurochemical and behavioral effects that overtop the ones previously observed by other authors. It is also important to note that, in addition to the discrepancy between our results and data from other laboratories using zebrafish models, numerous clinical and preclinical rodent studies support our findings: several authors described 5-HT tone as a general inhibitor of responsiveness, which decreases in response to sudden environmental changes allowing the expression of adequate behavioral responses. From this perspective, 5-HT acts as a context-dependent modulator that levels responsiveness into nonimpulsive ranges (Brodie and Shore, 1957; Depue and Spoont, 1986; Soubrié, 1986; Spoont, 1992; Lucki, 1998). Behrendt (2011) suggests that 5-HT downregulates responsivity partially through its actions on amygdala function (which theory is supported by several human fMRI studies investigating the mechanisms of selective 5-HT reuptake inhibitors in aversive contexts) (Del-Ben et al., 2005; Harmer et al., 2006; Murphy et al., 2009). In addition, isolation-induced enhanced serotonergic activity and fiber density in the amygdala were also reported in most rodent neurochemistry studies (Lehmann et al., 2003; Gos et al., 2006; Günther et al., 2008), in line with our current results.

In conclusion, we suggest that 5-HT tone of zebrafish restricts behavior under resting conditions, while its phasic decrease yields behavioral stress responses during a challenge. Social isolation during an adaptive time window leads to an abnormal 5-HT phenotype in juveniles, with serotonin levels inversely reflecting those of socially reared ones, thus exerting shifted responsivity as well. The phenomenon is modulated in the caudal recess of the telencephalon, where homolog structures of the mammalian amygdala and hippocampus are located. Our findings indicate that zebrafish possess a conserved serotonergic mechanism that context-dependently modulates environmental reactivity and is highly sensitive to early life experiences.

\section{References}

Aliczki M, Zelena D, Mikics E, Varga ZK, Pinter O, Bakos NV, Varga J, Haller J (2013) Monoacylglycerol lipase inhibition-induced changes in plasma corticosterone levels, anxiety and locomotor activity in male CD1 mice. Horm Behav 63:752-758.

Arakawa H (2007) Ontogeny of sex differences in defensive burying behavior in rats: effect of social isolation. Aggress Behav 33:38-47.

Arakawa H (2005) Interaction between isolation rearing and social development on exploratory behavior in male rats. Behav Processes 70:223-234.

Barba-Escobedo PA, Gould GG (2012) Visual social preferences of lone zebrafish in a novel environment: strain and anxiolytic effects. Genes Brain Behav 11:366-373.

Behrendt RP (2011) Neuroanatomy of social behaviour. An evolutionary and psychoanalytic perspective. London:Karnac Books.

Blaser RE, Peñalosa YM (2011) Stimuli affecting zebrafish (Danio rerio) behavior in the light/dark preference test. Physiol Behav 104:831-837.

Booij L, Tremblay RE, Szyf M, Benkelfat C (2015) Genetic and early environmental influences on the serotonin system: consequences for brain development and risk for psychopathology. J Psychiatry Neurosci 40:5-18.

Brodie BB, Shore PA (1957) A concept for a role of serotonin and norepinephrine as chemical mediators in the brain. Ann NY Acad Sci 66:631642. 
Chapman DP, Whitfield CL, Felitti VJ, Dube SR, Edwards VJ, Anda RF (2004) Adverse childhood experiences and the risk of depressive disorders in adulthood. J Affect Disord 82:217-225.

Chen F, Chen S, Liu S, Zhang C, Peng G (2015) Effects of lorazepam and WAY-200070 in larval zebrafish light/dark choice test. Neuropharmacology 95:226-233.

Del-Ben CM, Deakin JF, McKie S, Delvai NA, Williams SR, Elliott R, Dolan M, Anderson IM (2005) The effect of citalopram pretreatment on neuronal responses to neuropsychological tasks in normal volunteers: an fMRI study. Neuropsychopharmacology 30:1724-1734.

Depue RA, Spoont MR (1986) A behavioral dimension of constraint. Ann NY Acad Sci 487:47-62.

Bates D (2015) Fitting linear mixed-effects models using $\{$ lme4\}. J Stat Softw 67:1-48.

Dreosti E, Lopes G, Kampff AR, Wilson SW (2015) Development of social behavior in young zebrafish. Front Neural Circuits 9:39.

Egan RJ, Bergner CL, Hart PC, Cachat JM, Canavello PR, Elegante MF, Elkhayat SI, Bartels BK, Tien AK, Tien DH, Mohnot S, Beeson E, Glasgow E, Amri H, Zukowska Z, Kalueff AV (2009) Understanding behavioral and physiological phenotypes of stress and anxiety in zebrafish. Behav Brain Res 205:38-44.

Einon DF, Morgan MJ (1977) A critical period for social isolation in the rat. Dev Psychobiol 10:123-132

Faraway JJ (2016) Extending the linear model with R: generalized linear, mixed effects and nonparametric regression models. Boca Raton, FL: CRC.

Fone KC, Porkess MV (2008) Behavioural and neurochemical effects of postweaning social isolation in rodents: relevance to developmental neuropsychiatric disorders. Neurosci Biobehav Rev 32:1087-1102.

Forsatkar MN, Safari O, Boiti C (2017) Effects of social isolation on growth, stress response, and immunity of zebrafish. Acta Ethol 20:255-261.

Geyer MA (1995) Serotonergic functions in arousal and motor activity. Behav Brain Res 73:31-35.

Giacomini AC, de Abreu MS, Koakoski G, Idalêncio R, Kalichak F, Oliveira TA, da Rosa JG, Gusso D, Piato AL, Barcellos LJG (2015) My stress, our stress: blunted cortisol response to stress in isolated housed zebrafish. Physiol Behav 139:182-187.

Gläscher J, Adolphs R (2003) Processing of the arousal of subliminal and supraliminal emotional stimuli by the human amygdala. J Neurosci 23:10274-10282.

Gobert A, Rivet JM, Cistarelli L, Millan MJ (1997) Buspirone enhances duloxetine- and fluoxetine-induced increases in dialysate levels of dopamine and noradrenaline, but not serotonin, in the frontal cortex of freely moving rats. J Neurochem 68:1326-1329.

Gobert A, Rivet JM, Cistarelli L, Melon C, Millan MJ (1999) Buspirone modulates basal and fluoxetine-stimulated dialysate levels of dopamine, noradrenaline and serotonin in the frontal cortex of freely moving rats: activation of serotonin $1 \mathrm{~A}$ receptors and blockade of $\alpha 2$-adrenergic receptors underlie its actions. Neuroscience 93:1251-1262.

Gos T, Becker K, Bock J, Malecki U, Bogerts B, Poeggel G, Braun K (2006) Early neonatal and postweaning social emotional deprivation interferes with the maturation of serotonergic and tyrosine hydroxylase-immunoreactive afferent fiber systems in the rodent nucleus accumbens, hippocampus and amygdala. Neuroscience 140:811-821.

Gould GG (2011) Aquatic light/dark plus maze novel environment for assessing anxious versus exploratory behavior in zebrafish (Danio rerio) and other small teleost fish. In: Zebrafish neurobehavioral protocols, neuromethods (Kalueff AV, Cachat JM, eds), pp 99-108. Totowa, NJ: Humana.

Gross C, Zhuang X, Stark K, Ramboz S, Oosting R, Kirby L, Santarelli L, Beck S, Hen R (2002) Serotonin1A receptor acts during development to establish normal anxiety-like behaviour in the adult. Nature 416:396-400.

Günther L, Liebscher S, Jähkel M, Oehler J (2008) Effects of chronic citalopram treatment on 5-HT1A and 5-HT2A receptors in group- and isolation-housed mice. Eur J Pharmacol 593:49-61.

Gutiérrez HC, Colanesi S, Cooper B, Reichmann F, Young AM, Kelsh RN, Norton WH (2019) Endothelin neurotransmitter signalling controls zebrafish social behaviour. Sci Rep 9:3040.

Hall FS (1998) Social deprivation of neonatal, adolescent, and adult rats has distinct neurochemical and behavioral consequences. Crit Rev Neurobiol 12:129-162.
Haller J, Harold G, Sandi C, Neumann ID (2014) Effects of adverse early-life events on aggression and anti-social behaviours in animals and humans. J Neuroendocrinol 26:724-738

Haller J, Schraaf JV, Kruk MR (2001) Deviant forms of aggression in glucocorticoid hyporeactive rats: a model for 'pathological' aggression? J Neuroendocrinol 13:102-107.

Harmer CJ, Mackay CE, Reid CB, Cowen PJ, Goodwin GM (2006) Antidepressant drug treatment modifies the neural processing of nonconscious threat cues. Biol Psychiatry 59:816-820.

Jing J, Gillette R, Weiss KR (2009) Evolving concepts of arousal: insights from simple model systems. Rev Neurosci 20:405-427.

Kuznetsova A, Brockhoff PB, Christensen RHB (2017) lmerTest Package: Tests in Linear Mixed Effects Models. Journal of Statistical Software 82:1-26.

Lau BY, Mathur P, Gould GG, Guo S (2011) Identification of a brain center whose activity discriminates a choice behavior in zebrafish. Proc Natl Acad Sci USA 108:2581-2586.

Lechin F, Dijs B, van der Jara H, Orozco B, Baez S, Benaim M, Lechin M, Lechin A (1998) Effects of buspirone on plasma neurotransmitters in healthy subjects. J Neural Transm (Vienna) 105:561-573.

Lehmann K, Lesting J, Polascheck D, Teuchert-Noodt G (2003) Serotonin fibre densities in subcortical areas: differential effects of isolated rearing and methamphetamine. Brain Res Dev Brain Res 147:143-152.

Lieschke PD, Currie GJ (2007) Animal models of human disease: zebrafish swim into view. Nat Rev Genet 8:353-367.

Loane C, Politis M (2012) Buspirone: what is it all about? Brain Res 1461:111-118.

Lovett-Barron M, Andalman AS, Allen WE, Vesuna S, Kauvar I, Burns VM, Deisseroth K (2017) Ancestral circuits for the coordinated modulation of brain state. Cell 171:1411-1423.e17.

Lucki I (1998) The spectrum of behaviors influenced by serotonin. Biol Psychiatry 44:151-162.

Lukkes JL, Summers CH, Scholl JL, Renner KJ, Forster GL (2009a) Early life social isolation alters corticotropin-releasing factor responses in adult rats. Neuroscience 158:845-855.

Lukkes JL, Watt MJ, Lowry CA, Forster GL (2009b) Consequences of postweaning social isolation on anxiety behavior and related neural circuits in rodents. Front Behav Neurosci 3:18.

MacPhail RC, Brooks J, Hunter DL, Padnos B, Irons TD, Padilla S (2009) Locomotion in larval zebrafish: influence of time of day, lighting and ethanol. Neurotoxicology 30:52-58.

MacRae CA, Peterson RT (2015) Zebrafish as tools for drug discovery. Nat Rev Drug Discov 14:721-731.

Maximino C, da Silva AW, Gouveia A, Herculano AM (2011) Pharmacological analysis of zebrafish (Danio rerio) scototaxis. Prog Neuropsychopharmacol Biol Psychiatry 35:624-631.

Maximino C, Lima MG, Araujo J, Oliveira KR, Herculano AM, Stewart AM, Kyzar EJ, Cachat J, Kalueff AV (2013a) The serotonergic system of zebrafish: genomics, neuroanatomy and neuropharmacology, Serotonin Biosynthesis, Regul Heal Implic (Hall FS ed), pp. 53-67. New York, NY: Nova Science.

Maximino C, Puty B, Benzecry R, Araújo J, Lima MG, de Jesus Oliveira Batista E, Renata de Matos Oliveira K, Crespo-Lopez ME, Herculano AM (2013b) Role of serotonin in zebrafish (Danio rerio) anxiety: relationship with serotonin levels and effect of buspirone, WAY 100635, SB 224289, fluoxetine and para-chlorophenylalanine (pCPA) in two behavioral models. Neuropharmacology 71:83-97.

McBurnett K, Lahey BB, Rathouz PJ, Loeber R (2000) Low salivary cortisol and persistent aggression in boys referred for disruptive behavior. Arch Gen Psychiatry 57:38-43.

Mikics E, Barsy B, Barsvári B, Haller J (2005) Behavioral specificity of nongenomic glucocorticoid effects in rats: effects on risk assessment in the elevated plus-maze and the open-field. Horm Behav 48:152-162.

Murphy SE, Norbury R, O'Sullivan U, Cowen PJ, Harmer CJ (2009) Effect of a single dose of citalopram on amygdala response to emotional faces. $\mathrm{Br} \mathrm{J}$ Psychiatry 194:535-540.

Nederhof E, Schmidt MV (2012) Mismatch or cumulative stress: toward an integrated hypothesis of programming effects. Physiol Behav 106:691700.

Noldus LP, Spink AJ, Tegelenbosch RA (2001) EthoVision: a versatile video tracking system for automation of behavioral experiments. Behav Res Methods Instrum Comput 33:398-414. 
Norton WH, Folchert A, Bally-Cuif L (2008) Comparative analysis of serotonin receptor (HTR1A/HTR1B families) and transporter (slc6a4a/b) gene expression in the zebrafish brain. J Comp Neurol 511:521-542.

Panula P, Chen YC, Priyadarshini M, Kudo H, Semenova S, Sundvik M, Sallinen V (2010) The comparative neuroanatomy and neurochemistry of zebrafish CNS systems of relevance to human neuropsychiatric diseases. Neurobiol Dis 40:46-57.

Parker MO, Millington ME, Combe FJ, Brennan CH (2012) Housing conditions differentially affect physiological and behavioural stress responses of zebrafish, as well as the response to anxiolytics. PLoS One 7:e34992.

Peroutka SJ (1988) 5-Hydroxytryptamine receptor subtypes: molecular, biochemical and physiological characterization. Trends Neurosci 11:496500.

R Core Team (2017) R: A language and environment for statistical computing: R foundation for statistical computing. Vienna: R Core Team.

Raleigh MJ, McGuire MT, Brammer GL, Yuwiler A (1984) Social and environmental influences on blood serotonin concentrations in monkeys. Arch Gen Psychiatry 41:405-410.

Santarelli S, Lesuis SL, Wang XD, Wagner KV, Hartmann J, Labermaier C, Scharf SH, Müller MB, Holsboer F, Schmidt MV (2014) Evidence supporting the match/mismatch hypothesis of psychiatric disorders. Eur Neuropsychopharmacol 24:907-918.

Schloerke B, Crowley J, Cook D, Briatte F, Marbach M, Thoen E, Elberg A, Larmarange J (2017) GGally: extension to "ggplot2.”. Available at https:// tinyurl.com/y8vt726g.

Schneider CA, Rasband WS, Eliceiri KW (2012) NIH Image to ImageJ: 25 years of image analysis. Nat Methods 9:671-675.

Shams S, Chatterjee D, Gerlai R (2015) Chronic social isolation affects thigmotaxis and whole-brain serotonin levels in adult zebrafish. Behav Brain Res 292:283-287.

Shams S, Amlani S, Buske C, Chatterjee D, Gerlai R (2017a) Developmental social isolation affects adult behavior, social interaction, and dopamine metabolite levels in zebrafish. Dev Psychobiol 60:43-56.

Shams S, Seguin D, Facciol A, Chatterjee D, Gerlai R (2017b) Effect of social isolation on anxiety-related behaviors, cortisol, and monoamines in adult zebrafish. Behav Neurosci 131:492-504
Shireen E, Haleem DJ (2005) Motor effects of buspirone: relationship with dopamine and serotonin in the striatum. J Coll Physicians Surg Pak 15:753-756.

Soubrié P (1986) Reconciling the role of central serotonin neurons in human and animal behavior. Behav Brain Sci 9:319-335.

Spoont MR (1992) Modulatory role of serotonin in neural information processing: implications for human psychopathology. Psychol Bull 112:330350.

Thompson RF, Spencer WA (1966) Habituation: a model phenomenon for the study of neuronal substrates of behavior. Psychol Rev 73:16-43.

Toth M, Mikics E, Tulogdi A, Aliczki M, Haller J (2011) Post-weaning social isolation induces abnormal forms of aggression in conjunction with increased glucocorticoid and autonomic stress responses. Horm Behav 60:28-36.

Tudorache C, ter Braake A, Tromp M, Slabbekoorn H, Schaaf MJ (2015) Behavioral and physiological indicators of stress coping styles in larval zebrafish. Stress 18:121-128.

van Goozen SH, Matthys W, Cohen-Kettenis PT, Gispen-de Wied C, Wiegant VM, van Engeland H (1998) Salivary cortisol and cardiovascular activity during stress in oppositional-defiant disorder boys and normal controls. Biol Psychiatry 43:531-539.

Vanyukov MM, Moss HB, Plail JA, Blackson T, Mezzich AC, Tarter RE (1993) Antisocial symptoms in preadolescent boys and in their parents: associations with cortisol. Psychiatry Res 46:9-17.

Varga ZK, Zsigmond Á, Pejtsik D, Varga M, Demeter K, Mikics É, Haller J, Aliczki M (2018) The swimming plus-maze test: a novel high-throughput model for assessment of anxiety-related behaviour in larval and juvenile zebrafish (Danio rerio). Sci Rep 8:16590.

Westerfield M (2000) The zebrafish book: a guide for the laboratory use of zebrafish. Eugene: University of Oregon Press.

Wulliman MF, Rupp B, Reichert H (2012) Neuroanatomy of the zebrafish brain: a topological atlas. Birkhäuser: Basel.

Xin N, Jiang Y, Liu S, Zhou Y, Cheng Y (2020) Effects of prednisolone on behavior and hypothalamic-pituitary-interrenal axis activity in zebrafish. Environ Toxicol Pharmacol 75:103325.

Yokogawa T, Hannan MC, Burgess HA (2012) The dorsal raphe modulates sensory responsiveness during arousal in zebrafish. J Neurosci 32:1520515215 . 